\title{
Anisotropic Wellbore Stability Analysis: Impact on Failure Prediction
}

\author{
Michinori Asaka ${ }^{1,2}$ (D) Rune Martin Holt ${ }^{1}$
}

Received: 9 October 2020 / Accepted: 16 October 2020 / Published online: 3 November 2020

(c) The Author(s) 2020

\begin{abstract}
Shale formations are the main source of borehole stability problems during drilling operations. Suboptimal predictions of borehole failure may partly be caused by neglecting the anisotropic nature of shales: Conventional wellbore stability analysis is based on borehole stresses computed from isotropic linear elasticity (Kirsch solution) with the assumption of no induced pore pressure. This is very convenient for a practical implementation but does not always work for shales. Here, anisotropic wellbore stability analysis was performed targeting an offshore gas field to investigate in particular the impact of elastic anisotropy on borehole failure predictions. Stress concentration around a circular borehole in anisotropic shale was calculated by the Amadei solutions, and induced pore pressure was obtained from the Skempton parameters based on anisotropic poroelasticity. Borehole failure regions and modes were then predicted using the effective stresses and those are apparently consistent with observations. A comparison with the conventional approach suggests the importance of accounting for elastic anisotropy: Predicted failure regions, modes, and also the associated mud weight limits can be completely different. This observation may have significant implications for other fields since shale often show strong elastic anisotropy.
\end{abstract}

Keywords Wellbore stability $\cdot$ Mud weight $\cdot$ Shale $\cdot$ Anisotropy $\cdot$ Poroelasticity

\section{List of symbols}

$\mu_{1}, \mu_{2}, \mu_{3}$

$l_{2}, l_{3}, l_{4}$

$\beta_{i j}(i, j=1,2, \ldots 6)$

$\varphi_{i}(i=1,2,3)$

$\sigma_{x, 0}, \sigma_{y, 0}, \sigma_{z, 0}, \tau_{y z, 0}, \tau_{x z, 0}, \tau_{x y, 0}$

$\sigma_{x}, \sigma_{y}, \sigma_{z}, \tau_{y z}, \tau_{x z}, \tau_{x y}$

$\sigma_{x, \mathrm{TI}}, \sigma_{y, \mathrm{TI}}, \sigma_{z, \mathrm{TI}}$ $s_{i j}(i, j=1,2, \ldots 6)$

Roots of the sixth order poly-

nomial equation

Characteristic equations

Reduced strain coefficients

Compliance tensor com-

ponents of the anisotropic medium (voigt notation)

Analytical functions to express the stress functions

In situ stress components

in the borehole coordinate

system

Normal and shear stresses

in the borehole coordinate system

Normal stresses in the coordinate system aligned with transverse isotropy

Michinori Asaka

michinori.asaka@inpex.co.jp

1 Norwegian University of Science and Technology (NTNU), Trondheim, Norway

2 INPEX Corporation, Tokyo, Japan

$q$
$a$
$\theta$
$\theta_{\mathrm{c}}$
$B_{i j}(i, j=1,2,3)$
$B_{\mathrm{V}}$
$B_{\mathrm{H}}$
$A_{\mathrm{s}}, B_{\mathrm{s}}$
$\theta_{\mathrm{b}}$
$s_{i j k l}^{\mathrm{d}}(i, j, k, l=1,2,3)$
$s_{i j k l}^{\mathrm{s}}(i, j, k, l=1,2,3)$
$c_{\mathrm{f}}$
$p_{\mathrm{f}}$

Well pressure

Borehole radius

Angular borehole position

Rotation angle for transformation to the coordinate system used in this study Components of Skempton $B$ Vertical (normal to bedding) component of Skempton $B$ Horizontal (parallel to bedding) component of Skempton $B$

Commonly used Skempton parameters to express induced pore pressure Angle between the symmetry axis and the maximum principal stress Compliance tensor components of the drained rock Compliance tensor components of the grain Fluid compressibility Porosity Induced pore pressure 


\begin{tabular}{|c|c|}
\hline$\Delta \sigma_{i j}(i, j=1,2,3)$ & $\begin{array}{l}\text { Components of total stress } \\
\text { change }\end{array}$ \\
\hline$\sigma_{1}^{\prime}$ & $\begin{array}{l}\text { Maximum effective principal } \\
\text { stress }\end{array}$ \\
\hline$\sigma_{3}^{\prime}$ & $\begin{array}{l}\text { Minimum effective principal } \\
\text { stress }\end{array}$ \\
\hline$C_{0}$ & $\begin{array}{l}\text { Uniaxial compressive } \\
\text { strength of intact rock }\end{array}$ \\
\hline$\varphi_{\mathrm{f}}$ & Friction angle of intact rock \\
\hline$\tau_{\mathrm{w}}$ & $\begin{array}{l}\text { Shear stress acting on the } \\
\text { plane of weakness (bedding) }\end{array}$ \\
\hline$\sigma_{\mathrm{w}}^{\prime}$ & $\begin{array}{l}\text { Effective normal stress act- } \\
\text { ing on the plane of weakness } \\
\text { (bedding) }\end{array}$ \\
\hline$S_{\mathrm{w}}$ & $\begin{array}{l}\text { Cohesion of the plane of } \\
\text { weakness (bedding) }\end{array}$ \\
\hline$\mu_{\mathrm{w}}$ & $\begin{array}{l}\text { Sliding friction coefficient } \\
\text { of the plane of weakness } \\
\text { (bedding) }\end{array}$ \\
\hline$\varepsilon$ & $\begin{array}{l}\text { Thomsen's anisotropy } \\
\text { parameter epsilon }\end{array}$ \\
\hline$\gamma$ & $\begin{array}{l}\text { Thomsen's anisotropy } \\
\text { parameter gamma }\end{array}$ \\
\hline$\delta$ & $\begin{array}{l}\text { Thomsen's anisotropy } \\
\text { parameter delta }\end{array}$ \\
\hline$C_{i j}(i, j=1,2,3,4,5,6)$ & $\begin{array}{l}\text { Stiffness tensor components } \\
\text { of the anisotropic medium } \\
\text { (voigt notation) }\end{array}$ \\
\hline$V_{\mathrm{p}}\left(0^{\circ}\right)$ & $\begin{array}{l}\text { P-wave velocity parallel to } \\
\text { the symmetry axis }\end{array}$ \\
\hline$V_{\mathrm{p}}\left(90^{\circ}\right)$ & $\begin{array}{l}\text { P-wave velocity orthogonal } \\
\text { to the symmetry axis }\end{array}$ \\
\hline$V_{\mathrm{SH}}\left(0^{\circ}\right)$ & $\begin{array}{l}\text { SH-wave velocity parallel to } \\
\text { the symmetry axis }\end{array}$ \\
\hline$V_{\mathrm{SH}}\left(90^{\circ}\right)$ & $\begin{array}{l}\text { SH-wave velocity orthogonal } \\
\text { to the symmetry axis }\end{array}$ \\
\hline
\end{tabular}

\section{Introduction}

Borehole instability resulting in tight hole or stuck pipe situations is a notoriously expensive and time-consuming problem during drilling operations. Solutions are provided by properly adjusting the mud weight, the mud composition and the well design (hole inclination and azimuth) to avoid collapse of the formation around the borehole. Shale is the predominant problem formation. Dealing with shale instabilities requires knowledge and understanding of the mechanical behaviour of shales and the stress field around the borehole. Collapse criteria are primarily based on rock failure criteria for brittle materials, assuming that borehole stresses are given by linear elasticity theory. Traditionally, these considerations implicitly assume isotropic formations.
Anisotropy is however an inherent characteristic of shale: Its mechanical response depends on the orientation of principal stresses with respect to the symmetry of the material (Fjaer et al. 2008). The origin of the anisotropy is always heterogeneities on a smaller scale than the volume under investigation, ranging from layered sequences of different rock types down to molecular configurations (Fjaer et al. 2008). Shales often show strong elastic anisotropy that originates from the alignment and platy habit of its constituent minerals, as underpinned by existing laboratory measurements (e.g. Vernik and Nur 1992; Wang 2002; Szewczyk et al. 2018; Lozovyi and Bauer 2019). Shale strength is also anisotropic, with less resistance for failure within the symmetry plane ("weak plane") than in other directions (e.g. Lee et al. 2012). Since shale constitutes most of the overburden in conventional oil and gas field worldwide, it is of general interest to assess the impact of anisotropy on subsurface evaluation.

Borehole drilling induces changes in the stress distribution around the hole, with magnitudes depending on well trajectory, in-situ stresses, well pressure, and rock properties. The stress distribution should be correctly estimated to avoid suboptimal choice of well trajectory and well pressure, which may lead to hole instability. A standard approach in the oil and gas industry is to use the classical Kirsch solution (Kirsch 1898; Hiramatsu and Oka 1962), which assumes the rock to be isotropic, and to ignore the pore pressure change induced by the stress change. The approach is widely used because it is convenient for practical implementation. Elastic anisotropy will however influence the pattern of the stress change around the hole. The fundamentals of the stress change around the hole in anisotropic rock formations was established by Lekhnitskii (1963). Amadei (1983) gave complete formulas to calculate the stress change based on the work by Lekhnitskii (1963). Karpfinger et al. (2011) presented a rigorous validation of the Amadei solution by comparing with the numerical model.

For low permeability rocks such as shales, undrained conditions remain valid for a certain time after initial drillout. Induced pore pressure caused by the stress change around borehole should therefore be considered (note that the induced pore pressure is also relevant for highly permeable rocks, but drained conditions are rapidly reached in this case). This necessitates the application of anisotropic poroelasticity; not only the stress concentration, but also the induced pore pressure is affected by elastic anisotropy. For example, pore pressure change is only caused by the mean stress change for isotropic elasticity, but for anisotropic elastic media shear stresses can induce volumetric strain and lead to pore pressure change. Cheng (1997) gave a thorough introduction to the anisotropic poroelasticity and showed that Skempton's $A$ and $B$ parameters should be generalized to a second order tensor to describe the pore pressure change in 
the anisotropic rock formations. Based on the formula provided by Cheng (1997), Raaen et al. (2019) showed examples in which the pore pressure change around the borehole is largely affected by elastic anisotropy. Holt et al. (2018a) investigated the applicability of the anisotropic poroelasticity on shales and found that anisotropic poroelasticity theory gives an adequate description of the static elastic behavior of shale samples.

In this paper, the impact of elastic and strength anisotropy on the wellbore failure prediction is investigated. Shales are assumed to be transverse isotropic material. Stress concentration around a circular borehole in anisotropic shale is calculated by the Amadei solutions, and induced pore pressure is obtained from the anisotropic poroelasticity. A similar approach was adopted by Kanfar et al. (2015a), in which optimum mud weight estimation was performed for both isotropic and anisotropic rocks; induced pore pressure was taken into account for both cases. Here, we compare the anisotropic approach (the Amadei solution + anisotropic poroelasticity) with the conventional approach based on the Kirsch equation and the assumption of no induced pore pressure, and demonstrate that not only optimum mud weight but also failure regions and modes predicted by the approaches can be completely different. Realistic input parameters were used targeting a particular field with strike-slip stress regime. Anisotropic rock properties estimation was performed using available information and its uncertainty will be discussed. Our focus is hence the initial poroelastic drilling effect, detailed comparison with the conventional approach, and practical application of the method using available information. Since shales usually contain weak planes such as beddings, two Mohr-Coulomb failure criteria are adopted, one for the intact rock and the other for the weak bedding plane. Predicted failures based on the anisotropic approach are apparently consistent with observations.

Impact of consolidation (e.g., Abousleiman and Cui 1998), temperature (e.g., Abousleiman and Ekbote 2005; Gao et al. 2017a, b; Ghassemi and Diek 2002), chemical interaction between shale and drilling fluid (e.g., Ekbote and Abousleiman 2006; Ghassemi and Diek 2003) are out of the scope of this study because practical implementation was found to be difficult in the target field. Measured downhole annulus temperature suggests that the initial temperature difference between the drilled rock and the drilling fluid is about $10^{\circ}-20^{\circ}$, but limited information makes it difficult to correctly assess the impact. For example, thermal osmosis effect could have significant impact on the induced pore pressure (Gao et al. 2017a; Ghassemi and Diek 2002), however, the thermo osmotic coefficient has not been measured in the field and reported values are limited (Soler 2001). Chemo-poromechanical properties of shales are also not commonly available (Ghassemi and Diek 2003). Moreover, those available analytical solutions for transversely isotropic material assume that the plane of isotropy always perpendicular to the borehole axis, and numerical model is required to remove the assumption (Kanfar et al. 2015b, 2016, 2017).

\section{Methods}

\subsection{Amadei Solution}

For any elastostatic problem, stress, strain and displacement components must satisfy the equations of equilibrium, equations of compatibility for strains, strain displacement relations, constitutive relations and the boundary conditions. Stress concentration around a circular borehole in anisotropic medium can be derived as follows [see Amadei (1983) for details]:

1. Combine two conditions of compatibility for strains and the constitutive relations to produce two relations between the stress components and the potential (those relations are often called the Beltrami Michell equations of compatibility).

2. Introduce two stress functions (these reduces the number of unknowns) and rewrite the two relations from step 1 as a system of two differential equations that the stress functions must satisfy.

3. Solve for the two stress functions according to the boundary conditions along the contour of the hole.

4. Derive stress from the solutions obtained in step 3 (the stress components can be expressed with the derivatives of two stress functions).

Regarding the step 3, Lekhnitskii (1963) proposed a solution of the system that satisfies the boundary conditions. To solve the system, the roots $\left(\mu_{i}[i=1,2,3]\right)$ of the sixth order polynomial equation $l_{2} l_{4}-l_{3}^{2}=0$ need to be derived, with $l_{2}$, $l_{3}$ and $l_{4}$ are the characteristic equations expressed as:

$l_{2}=\beta_{44}-2 \beta_{45} \mu+\beta_{55} \mu^{2}$

$l_{3}=-\beta_{24}+\left(\beta_{25}+\beta_{46}\right) \mu-\left(\beta_{14}+\beta_{56}\right) \mu^{2}+\beta_{15} \mu^{3}$

$l_{4}=-\beta_{22}-2 \beta_{26} \mu+\left(2 \beta_{12}+\beta_{66}\right) \mu^{2}-2 \beta_{16} \mu^{3}+\beta_{11} \mu^{4}$,

where $\beta_{i j}$ is the reduced strain coefficients defined as:

$\beta_{i j}=s_{i j}-\frac{s_{i 3} s_{j 3}}{s_{33}}, \quad(i, j=1,2, \ldots 6)$,

$s_{i j}(i, j=1,2, \ldots 6)$ are the compliance tensor components of the anisotropic medium. The stress functions can then be expressed in terms of the roots and analytical functions $\left(\varphi_{i}[i=1,2,3]\right.$ in below equations) which need to be solved with the boundary conditions. The derivatives of two stress 
functions (step 4 above) result in the expressions for the stress components:

$\sigma_{x}=2 \operatorname{Re}\left[\mu_{1}^{2} \varphi_{1}^{\prime}\left(z_{1}\right)+\mu_{2}^{2} \varphi_{2}^{\prime}\left(z_{2}\right)+\lambda_{3} \mu_{3}^{2} \varphi_{3}^{\prime}\left(z_{3}\right)\right]$

$\sigma_{y}=2 \operatorname{Re}\left[\varphi_{1}^{\prime}\left(z_{1}\right)+\varphi_{2}^{\prime}\left(z_{2}\right)+\lambda_{3} \varphi_{3}^{\prime}\left(z_{3}\right)\right]$

$\tau_{y z}=-2 \operatorname{Re}\left[\lambda_{1} \varphi_{1}^{\prime}\left(z_{1}\right)+\lambda_{2} \varphi_{2}^{\prime}\left(z_{2}\right)+\varphi_{3}^{\prime}\left(z_{3}\right)\right]$

$\tau_{x z}=2 \operatorname{Re}\left[\lambda_{1} \mu_{1} \varphi_{1}^{\prime}\left(z_{1}\right)+\lambda_{2} \mu_{2} \varphi_{2}^{\prime}\left(z_{2}\right)+\mu_{3} \varphi_{3}^{\prime}\left(z_{3}\right)\right]$

$\tau_{x y}=-2 \operatorname{Re}\left[\mu_{1} \varphi_{1}^{\prime}\left(z_{1}\right)+\mu_{2} \varphi_{2}^{\prime}\left(z_{2}\right)+\lambda_{3} \mu_{3} \varphi_{3}^{\prime}\left(z_{3}\right)\right]$,

which should be added to the far field in situ stress components $\left(\sigma_{x, 0}, \sigma_{y, 0}, \tau_{y z, 0}, \tau_{x z, 0}\right.$ and $\left.\tau_{x y, 0}\right)$ to obtain the total stress components. The analytical functions $\left(\varphi_{i}[i=1,2,3]\right)$, which need to be solved with the boundary conditions, satisfies: coordinate system is different from the traditional top-ofhole borehole coordinate system where the $x$-axis points to the upward direction in the cross-sectional plane. The difference should be accounted for to correctly display the stress calculation results). For isotropy and transverse isotropy with the borehole along the unique axis or normal to the unique axis, the polynomial $l_{3}$ is identical to zero. The roots hence have to be derived from the other polynomials, two from $l_{4}$ and one from $l_{2}$. As suggested by Raaen et al. (2019), $\mu_{1}$ and $\mu_{2}$ were defined as the solution of $l_{4}=0$, while $\mu_{3}$ was defined as the solution of $l_{2}=0$.

$\sigma_{z}$ can be obtained based on the plane strain assumption:

$\varphi_{1}^{\prime}\left(z_{1}\right)=\frac{-1}{a \Delta \sqrt{\left(\frac{z_{1}}{a}\right)^{2}-1-\mu_{1}^{2}}}\left\{\left(\mu_{2}-\mu_{3} \lambda_{2} \lambda_{3}\right) \overline{a_{1}}+\left(\lambda_{2} \lambda_{3}-1\right) \overline{b_{1}}+\lambda_{3}\left(\mu_{3}-\mu_{2}\right) \overline{c_{1}}\right\} / \xi_{1}$

$\varphi_{2}^{\prime}\left(z_{2}\right)=\frac{-1}{a \Delta \sqrt{\left(\frac{z_{2}}{a}\right)^{2}-1-\mu_{2}^{2}}}\left\{\left(\lambda_{1} \lambda_{3} \mu_{3}-\mu_{1}\right) \overline{a_{1}}+\left(1-\lambda_{1} \lambda_{3}\right) \overline{b_{1}}+\lambda_{3}\left(\mu_{1}-\mu_{3}\right) \overline{c_{1}}\right\} / \xi_{2}$

$\varphi_{3}^{\prime}\left(z_{3}\right)=\frac{-1}{a \Delta \sqrt{\left(\frac{z_{3}}{a}\right)^{2}-1-\mu_{3}^{2}}}\left\{\left(\mu_{1} \lambda_{2}-\mu_{2} \lambda_{1}\right) \overline{a_{1}}+\left(\lambda_{1}-\lambda_{2}\right) \overline{b_{1}}+\left(\mu_{2}-\mu_{1}\right) \overline{c_{1}}\right\} / \xi_{3}$,

with

$\xi_{i}=\frac{\frac{z_{i}}{a}+\sqrt{\left(\frac{z_{i}}{a}\right)^{2}-1-\mu_{i}^{2}}}{1-i \mu_{i}}$

$\Delta=\mu_{2}-\mu_{1}+\lambda_{2} \lambda_{3}\left(\mu_{1}-\mu_{3}\right)+\lambda_{1} \lambda_{3}\left(\mu_{3}-\mu_{2}\right)$

$\overline{a_{1}}=-\frac{a}{2}\left(\sigma_{y, 0}-i \tau_{x y, 0}\right)+\frac{q a}{2}$

$\overline{b_{1}}=\frac{a}{2}\left(\tau_{x y, 0}-i \sigma_{x, 0}\right)+\frac{i q a}{2}$

$\overline{c_{1}}=\frac{a}{2}\left(\tau_{y z, 0}-i \tau_{x z, 0}\right)$,

$q$ is well pressure and $a$ is borehole radius. Note that the complex variable $z_{i}=x+\mu_{i} y=a \times \cos \theta+\mu_{i} \times a \times \sin \theta$ is a function of angular borehole position $\theta$. The complex variables $\lambda_{i}(i=1,2,3)$ are defined as:

$\lambda_{1}=-\frac{l_{3}\left(\mu_{1}\right)}{l_{2}\left(\mu_{1}\right)}, \lambda_{2}=-\frac{l_{3}\left(\mu_{2}\right)}{l_{2}\left(\mu_{2}\right)}, \lambda_{3}=-\frac{l_{3}\left(\mu_{3}\right)}{l_{4}\left(\mu_{3}\right)}$.

Fang (2018) proposed a coordinate system whose y-axis lies in the plane of isotropy where the stiffness matrix can be simplified to have at most thirteen non-zero components, which reduces coefficients of the sixth order polynomial equation. The stress calculation was hence performed in the coordinate system in this paper (It should be noted that the $\sigma_{z}=-\frac{1}{s_{33}}\left(s_{31} \sigma_{x}+s_{32} \sigma_{y}+s_{34} \tau_{y z}+s_{35} \tau_{x z}+s_{36} \tau_{x y}\right)$,

which should be added to the far field in situ stress component $\left(\sigma_{z, 0}\right)$ to obtain the total stress.

As mentioned above, the stress calculation was performed in the coordinate system proposed by Fang (2018). In-situ stresses and the material stiffness matrix were therefore transformed to the coordinate system by conducting three rotations: first to the global coordinate system; second to the traditional top-of-hole borehole coordinate system according to the well azimuth and inclination; third to the coordinate system proposed by Fang (2018). The third transformation is a rotation about the $z$-axis by an angle $\theta_{c}$, which is determined from the stiffness matrix. See Fang (2018) for the derivation of $\theta_{\mathrm{c}}$. Verification of the stress calculation was performed by reproducing the results of Karpfinger et al. (2011).

\subsection{Anisotropic Poroelasticity}

Induced pore pressure around a circular borehole in anisotropic shale was calculated using anisotropic Skempton $B$ parameters. The stress concentration based on the Amadei 
solution was used as an input stress for the calculation. Cheng (1997) gave the following formula for Skempton $B$ in an anisotropic porous medium:

$B_{i j}=\frac{3\left(s_{i j k k}^{\mathrm{d}}-s_{i j k k}^{\mathrm{s}}\right)}{A}$,

with

$A=\left(s_{i i j j}^{\mathrm{d}}-s_{i i j j}^{\mathrm{s}}\right)+\Phi\left(c_{\mathrm{f}}-a_{i i}^{\mathrm{s}}\right)$.

If we assume that the skeleton of the porous material is homogeneous at the pore (microscopic) scale, $a_{i i}^{\mathrm{s}}=s_{i i k k}^{\mathrm{s}}$. Skempton parameters are therefore defined by the compliance of the drained skeleton $s_{i j k l}^{\mathrm{d}}$, and that of the grain $s_{i j k l}^{\mathrm{s}}$, the fluid compressibility $c_{\mathrm{f}}$, and porosity $\Phi$.

For transverse isotropy aligned with the coordinate system, $B_{i j}$ will be diagonal (Raaen et al. 2019), which simplifies the induced pore pressure calculations as follows:

$\Delta p_{\mathrm{f}}=\frac{1}{3} B_{i j} \Delta \sigma_{i j}=\frac{1}{3}\left\{B_{\mathrm{V}} \Delta \sigma_{z, \mathrm{TI}}+B_{\mathrm{H}}\left(\Delta \sigma_{x, \mathrm{TI}}+\Delta \sigma_{y, \mathrm{TI}}\right)\right\}$,

where $\sigma_{x, \mathrm{TI}}, \sigma_{y, \mathrm{TI}}$, and $\sigma_{z, \mathrm{TI}}$ are normal stresses in the coordinate system aligned with transverse isotropy. The induced pore pressure calculation was therefore conducted in the coordinate system aligned with transverse isotropy; In-situ stresses and stresses calculated using the Amadei solutions were transformed to the coordinate system by performing three rotations: first to the traditional top-of-hole borehole coordinate system; second to the global coordinate system; third to the coordinate system aligned with transverse isotropy.

Note that the commonly used Skempton parameters $A_{\mathrm{s}}$ and $B_{\mathrm{s}}$ relate to $B_{\mathrm{V}}$ and $B_{\mathrm{H}}$ as (e.g. Holt et al. 2018b):

$$
\begin{aligned}
B_{\mathrm{s}} & =\frac{B_{\mathrm{V}}+2 B_{\mathrm{H}}}{3} \\
A_{\mathrm{s}}\left(\theta_{\mathrm{b}}\right) & =\frac{B_{\mathrm{V}} \cos ^{2} \theta_{\mathrm{b}}+B_{\mathrm{H}} \sin ^{2} \theta_{\mathrm{b}}}{3 B_{\mathrm{s}}},
\end{aligned}
$$

where $\theta_{\mathrm{b}}$ is the angle between the symmetry axis and the maximum principal stress. Verification of the induced pore pressure calculation was performed by reproducing the results of Raaen et al. (2019).

\subsection{Failure Criteria}

Since shales usually contain weak planes such as beddings, two Mohr-Coulomb failure criteria are adopted; shear failure across the intact rock and the slippage along the weak bedding planes. Lee et al. (2012) used the similar approach. The Mohr-Coulomb failure criterion for the intact rock is expressed as (Fjaer et al. 2008):
$\sigma_{1}^{\prime}=C_{0}+\sigma_{3}^{\prime} \tan ^{2} \beta$

where $\sigma_{1}^{\prime}$ and $\sigma_{3}^{\prime}$ are the maximum and minimum effective principal stresses, respectively. $C_{0}$ is the uniaxial compressive strength and $\beta=\pi / 4+\varphi_{\mathrm{f}} / 2$ ( $\varphi_{\mathrm{f}}$ is the friction angle). Jaeger and Cook (1979) gave the following formula for the Mohr-Coulomb failure criterion for the weak bedding planes:

$\tau_{\mathrm{w}}=S_{\mathrm{w}}+\mu_{\mathrm{w}} \sigma_{\mathrm{w}}^{\prime}$,

where $\tau_{\mathrm{w}}$ and $\sigma_{\mathrm{w}}^{\prime}$ are the shear and effective normal stresses acting on the plane of weakness (bedding), respectively. $S_{\mathrm{w}}$ and $\mu_{\mathrm{w}}$ are the cohesion and the sliding friction coefficient of the plane of weakness, respectively. Stresses have to be projected onto the surface of bedding plane by stress transformation to obtain the shear and effective normal stresses.

Tensile failure was also considered, which takes place when the effective tensile stress at the borehole wall is equal to the tensile strength $T$ of the rock. Note that the Terzaghi effective stress was used for failure predictions since it is generally accepted that compressive failure, as well as tensile failure, is controlled by the Terzaghi effective stress (Cornet and Fairhurst 1974; Fjaer et al. 2008; Bouteca and Gueguen 1999).

\section{Wellbore Stability Analysis}

The wellbore failure prediction was conducted targeting a particular field. The field is located within the Browse Basin on the North West Shelf of Australia, which is considered to be one of the most prolific areas in terms of hydrocarbon accumulation in Australia. The input parameters are listed in Table 1. As shown in the table, in situ stress state is a strikeslip regime with significant stress anisotropy (note that the maximum and minimum horizontal stresses correspond to approximately 73 and $54 \mathrm{MPa}$, respectively) (Asaka et al. 2016). The target interval is a thick shale interval with strong elastic anisotropy. Here, Thomsen's anisotropy parameters (Thomsen 1986) were used to describe the elastic anisotropy of shale since it is useful for conveniently characterizing the elastic constants of a transversely isotropic elastic medium. For weak anisotropy, anisotropy parameter epsilon can be seen to describe the fractional difference between the P-wave velocities parallel and orthogonal to the symmetry axis (Mavko et al. 2009):

$\varepsilon=\frac{C_{11}-C_{33}}{2 C_{33}} \approx \frac{V_{\mathrm{p}}\left(90^{\circ}\right)-V_{\mathrm{p}}\left(0^{\circ}\right)}{V_{\mathrm{p}}\left(0^{\circ}\right)}$. 
Table 1 Input parameters for the wellbore stability analysis

\begin{tabular}{ll}
\hline Well depth $(\mathrm{m})$ & 3100 \\
Overburden stress $\left(\mathrm{g} / \mathrm{cm}^{3}\right)$ & 2.0 \\
Maximum horizontal stress $\left(\mathrm{g} / \mathrm{cm}^{3}\right)$ & 2.39 \\
Minimum horizontal stress $\left(\mathrm{g} / \mathrm{cm}^{3}\right)$ & 1.77 \\
Maximum horizontal direction & $\mathrm{N} 90^{\circ} \mathrm{E}$ \\
Pore pressure $\left(\mathrm{g} / \mathrm{cm}^{3}\right)$ & 1.5 \\
Mud weight $\left(\mathrm{g} / \mathrm{cm}^{3}\right)$ & 1.63 \\
UCS of rock matrix $(\mathrm{MPa})$ & 55 \\
Coefficient of internal friction of rock matrix & 0.6 \\
Tensile strength of rock matrix $(\mathrm{MPa})$ & 0 \\
Porosity & 0.2 \\
Density $\left(\mathrm{g} / \mathrm{cm}^{3}\right)$ & 2.42 \\
Vertical P-wave velocity of undrained rock $(\mathrm{km} / \mathrm{s})$ & 2.41 \\
Vertical S-wave velocity of undrained rock $(\mathrm{km} / \mathrm{s})$ & 1.1 \\
Anisotropy parameter delta of undrained rock & 0.12 \\
Anisotropy parameter epsilon of undrained rock & 0.21 \\
Anisotropy parameter gamma of undrained rock & 0.6 \\
Undrained rock Young's modulus for loading normal to bedding $(\mathrm{GPa})$ & 6.99 \\
Undrained rock Young's modulus for loading parallel to bedding $(\mathrm{GPa})$ & 13.16 \\
Out-of-plane rock shear modulus $(\mathrm{GPa})$ & 2.93 \\
Undrained rock Poisson's ratio for loading normal to bedding & 0.36 \\
Undrained rock Poisson's ratio for loading parallel to bedding & 0.02 \\
Grain bulk modulus $(\mathrm{GPa})$ & 25 \\
Fluid bulk modulus $(\mathrm{GPa})$ & 2.6 \\
Cohesion of weak plane & 2 \\
Sliding friction coefficient of weak plane & 0.5 \\
Dip angle of weak plane $\left({ }^{\circ}\right)$ & 0 \\
\hline
\end{tabular}

Epsilon therefore describes what is usually called the "P-wave anisotropy". Similarly, for weak anisotropy, gamma can be seen to describe the fractional difference between the $\mathrm{SH}$-wave velocities parallel and orthogonal to the symmetry axis, which is equivalent to the difference between velocities of S-waves polarized parallel and normal to the symmetry axis, both propagating normal to the symmetry axis (Mavko et al. 2009):

$\gamma=\frac{C_{66}-C_{44}}{2 C_{44}} \approx \frac{V_{\mathrm{SH}}\left(90^{\circ}\right)-V_{\mathrm{SH}}\left(0^{\circ}\right)}{V_{\mathrm{SH}}\left(0^{\circ}\right)}$.

The physical meaning of delta is not as clear as epsilon and gamma, but it is an important parameter affecting the small-offset normal moveout velocity and offset-dependent reflection amplitudes:

$\delta=\frac{\left(C_{13}+C_{44}\right)^{2}-\left(C_{33}-C_{44}\right)^{2}}{2 C_{33}\left(C_{33}-C_{44}\right)}$.

For example, the normal moveout velocity can be expressed as $V_{\mathrm{p}}\left(0^{\circ}\right) \sqrt{1+2 \delta}$ (Thomsen 1986). Elastic properties of undrained shale are based on log data except for anisotropy parameters delta and epsilon; those are estimated mainly from seismic pre-stack depth migration velocity. Anisotropy parameter gamma was estimated from the Stoneley wave velocity measured by sonic tools. Stoneley wave gives $C_{66}$ because its low-frequency asymptote coincides with the tube wave speed, which is a function of $C_{66}$ (Norris and Sinha 1993).

Fluid bulk modulus was estimated using Batzle and Wang equations (Batzle and Wang 1992). Skempton's parameters have not been measured in this field, and they therefore need to be estimated. Grain bulk modulus is necessary for the estimation and is assumed to be isotropic with a value of $25 \mathrm{GPa}$. This value is based on clay velocity interpreted by extrapolating empirical relations (Castagna et al. 1993) and similar to the estimated value based on anisotropic poroelasticity by Holt et al. (2018a). With the fluid bulk modulus, grain bulk modulus, undrained rock properties, and porosity, Skempton's parameters can be calculated using the inverse anisotropic Gassmann equation (Mavko et al. 2009) and Eqs. (8) and (9). The applicability of this procedure might be challenged, however, Holt et al. (2018a) found that anisotropic poroelasticity theory based on Gassmann's equations adequately describe static behavior of shale in 
Table 2 Calculated Skempton's parameters and Biot coefficients

\begin{tabular}{ll}
\hline$B_{\mathrm{H}}$ & 0.41 \\
$B_{\mathrm{V}}$ & 1.14 \\
$A_{\mathrm{s}}(0)$ & 0.58 \\
$B_{\mathrm{s}}$ & 0.65 \\
$\alpha_{\mathrm{H}}$ & 0.73 \\
$\alpha_{\mathrm{V}}$ & 0.79 \\
\hline
\end{tabular}

terms of undrained vs drained moduli, Skempton's parameters and Biot coefficients. The calculated parameters are listed in Table 2. Skempton's parameters show significant anisotropy in which the value in the direction perpendicular to bedding is much larger than that in the direction parallel to bedding. This means that stress change in the direction perpendicular to bedding has much larger impact on the induced pore pressure than that in the direction parallel to bedding. The induced pore pressure in anisotropic elastic rock is therefore different from the isotropic elastic rock in which the pore pressure change is zero under constant mean stress conditions. Actual input mud weight is used in the existing inclined well, which was drilled in the maximum horizontal stress direction.

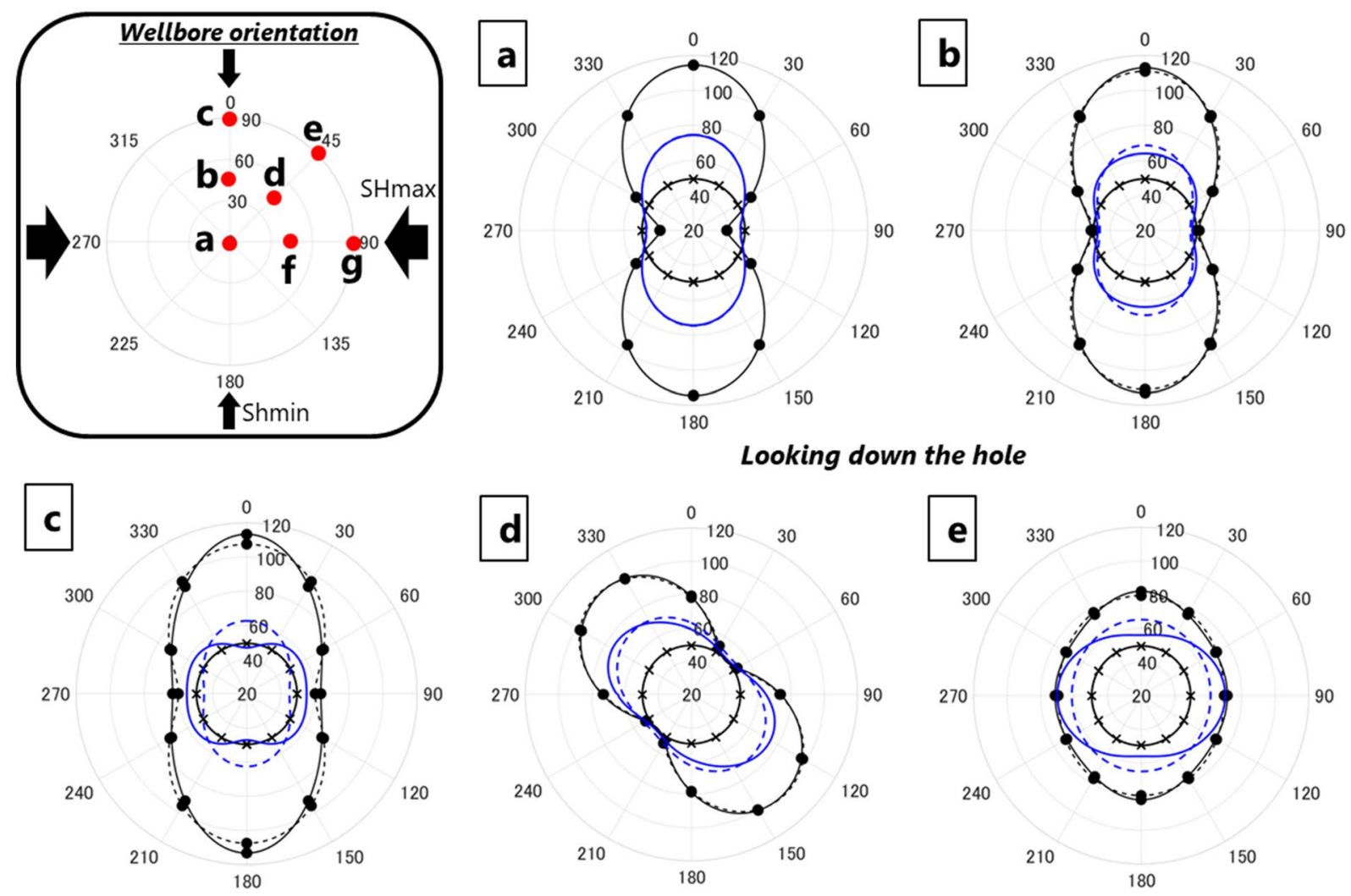

Looking down the hole
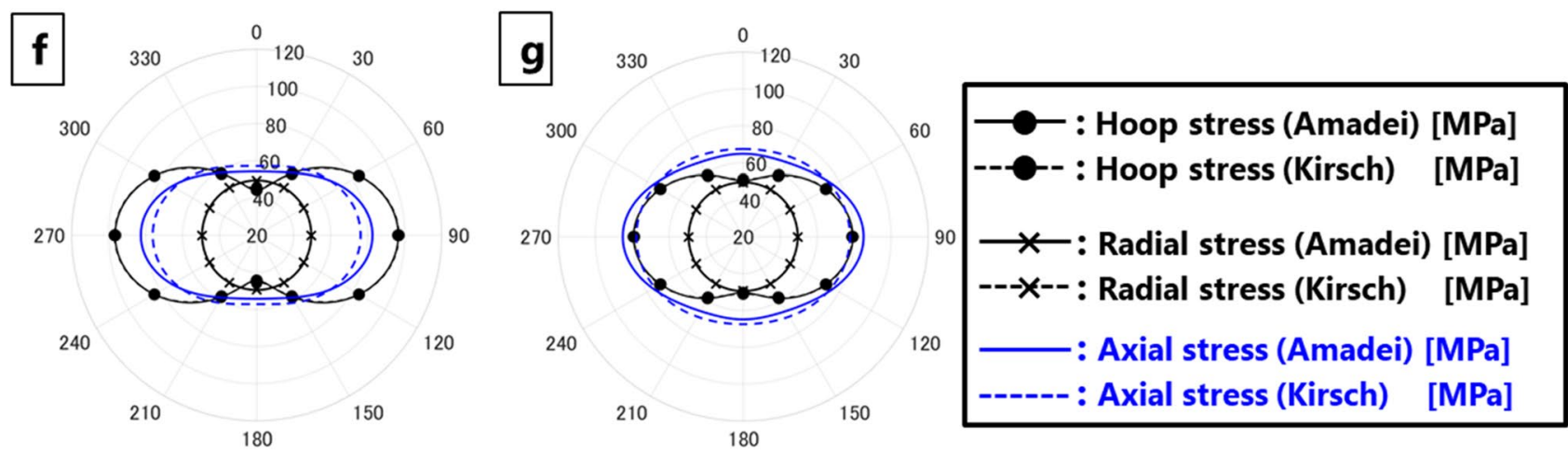

Fig. 1 A comparison of stress concentration at a circular borehole wall based on the anisotropic approach (the Amadei solution) with that based on the isotropic approach (the Kirsch solution) for seven different wellbore orientations (view: looking down the borehole) 


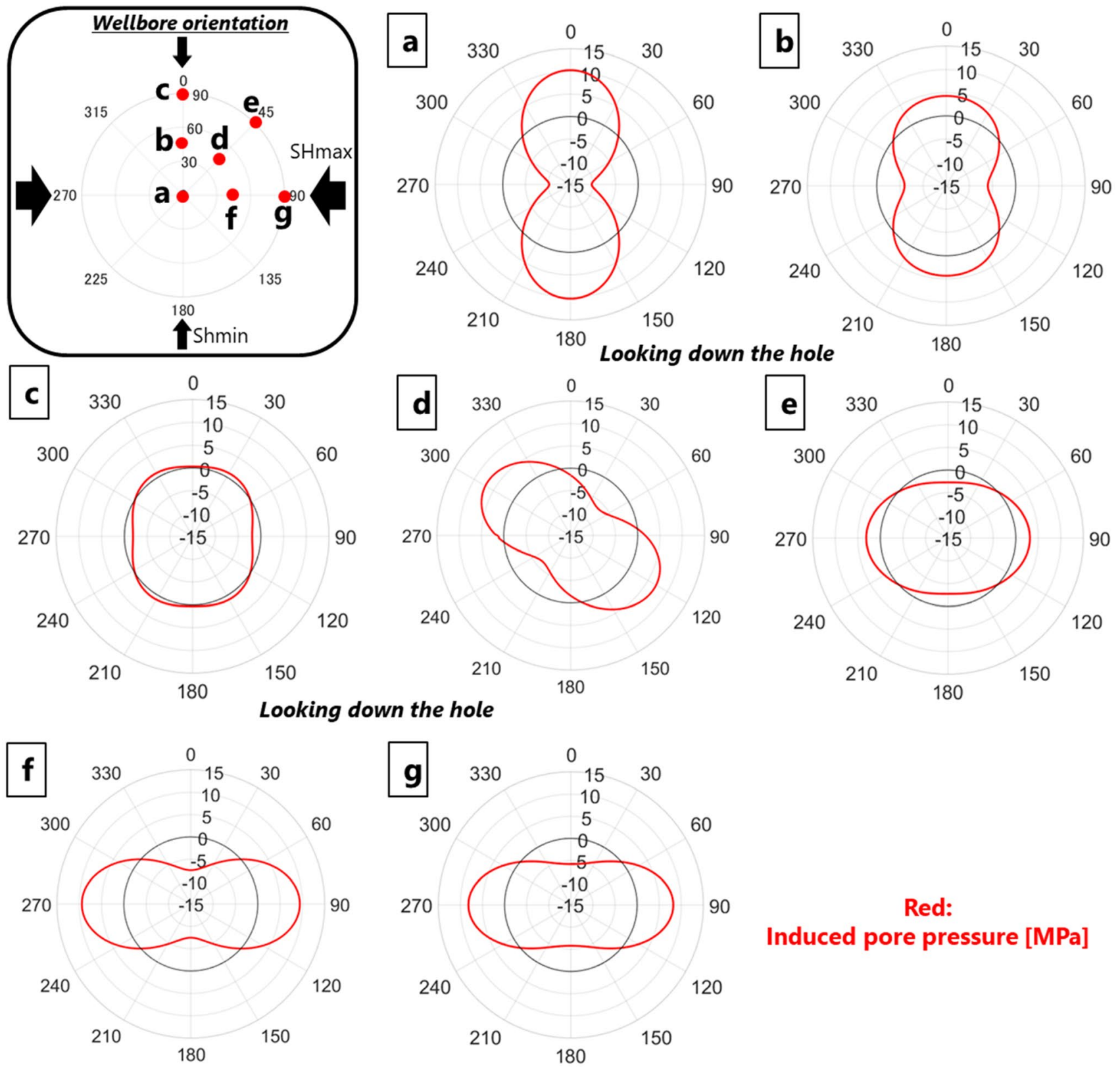

Fig. 2 Induced pore pressure at a circular borehole wall based on the anisotropic approach (the Amadei solution + anisotropic poroelasticity) for seven different wellbore orientations

First, results of wellbore stability analysis using the parameters listed in Table 1 will be shown in this section. Then, uncertainty related to Skempton's parameters will be discussed in the next section, where different undrained rock anisotropy parameters and grain properties are tested.

Figure 1 shows a comparison between the total stress at the borehole wall based on the Amadei solution and that based on the Kirsch solution for seven different wellbore orientations. Note that undrained elastic properties were used for all total stress calculations in this paper since our focus is the initial poroelastic drilling effect. As shown in the figure, the axial stress is different especially for the orientation c, in which a horizontal well is drilled towards the minimum horizontal stress direction. The axial stress becomes the minimum stress among the three stresses shown here at top and bottom of the borehole while it becomes higher than the radial stress at sides of the borehole for the anisotropic case. The impact of anisotropy on the hoop stress is not significant in all orientations considered here.

Figure 2 shows the induced pore pressure based on the stress calculated from the Amadei solution and anisotropic 


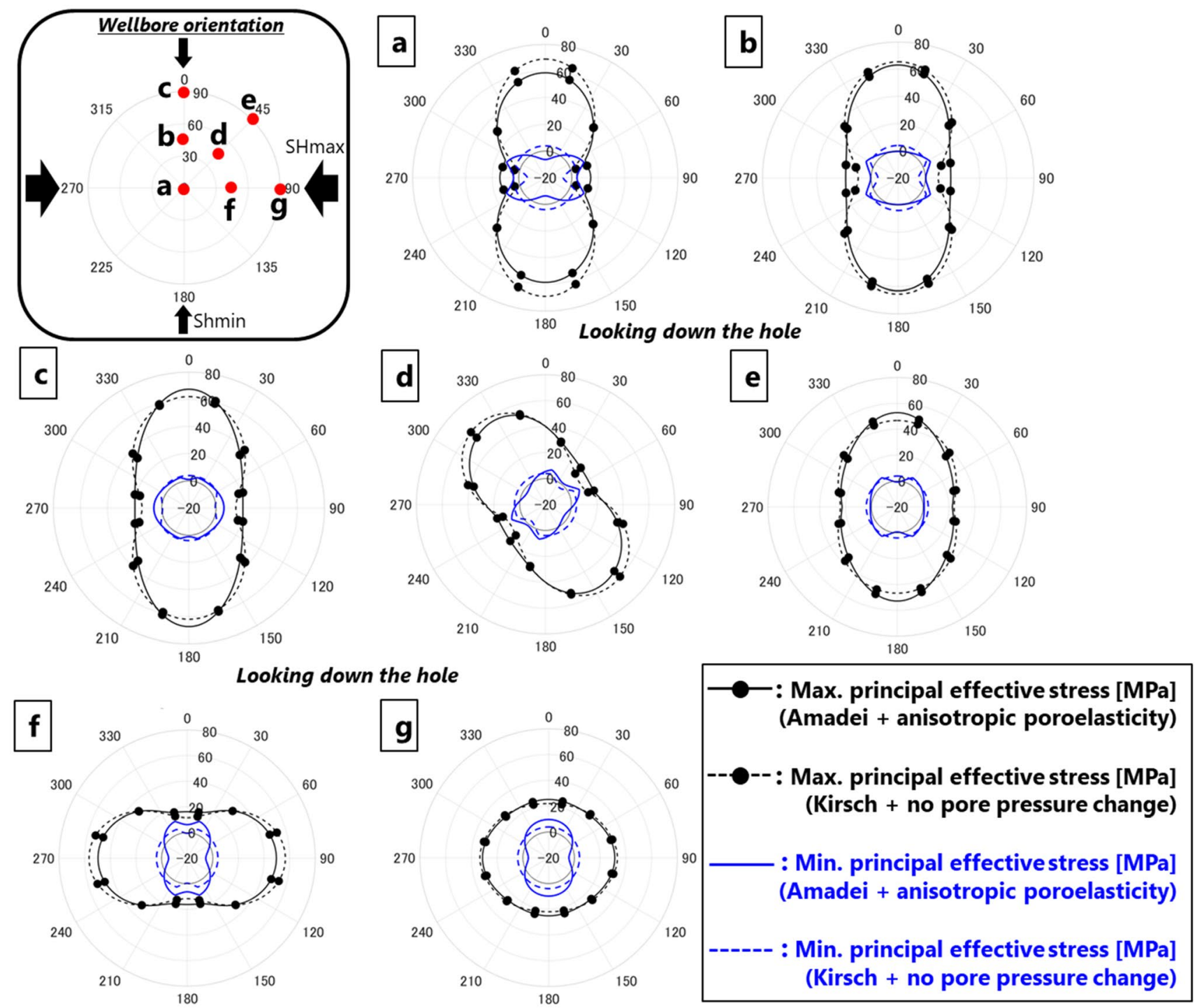

Fig. 3 A comparison of the minimum and maximum effective stresses at a circular borehole wall based on the anisotropic approach (the Amadei solution + anisotropic poroelasticity) with that based on the conventional approach (the Kirsch solution + no induced pore pressure assumption) for seven different wellbore orientations

poroelasticity for the 7 wellbore orientations. As shown in the figure, the position and the magnitude of the induced pore pressure strongly depend on the wellbore orientation. Wellbore orientation c shows small induced pore pressure. This is because of the aforementioned anisotropy in Skempton's parameters; induced pore pressure is less sensitive to changes in stress parallel to bedding.

Figure 3 shows a comparison of the minimum and maximum principal effective stresses based on the anisotropic approach (the Amadei solution + anisotropic poroelasticity) and that based on the conventional approach (the Kirsch solution + no induced pore pressure assumption). The position of the negative principal effective stress is completely different. For example, the conventional approach shows large negative values at sides of borehole for the orientation a, but the minimum principal effective stress given by the anisotropic approach is not negative at the location; instead, it is negative at top and bottom of borehole (note that, for the orientation a, the top and the bottom correspond to North and South, respectively). This is because of pore pressure reduction at sides and pore pressure increase at top and bottom of borehole as shown in Fig. 2. Similarly, the anisotropic approach gives negative principal effective stress at sides of borehole for wellbore orientations $f$ and $g$. The negative principal effective stress is caused by the negative radial effective stress, which may trigger the radial tensile failure (tensile failures throughout the wellbore circumference) (Fjaer et al. 2008; Skea et al. 2018). 


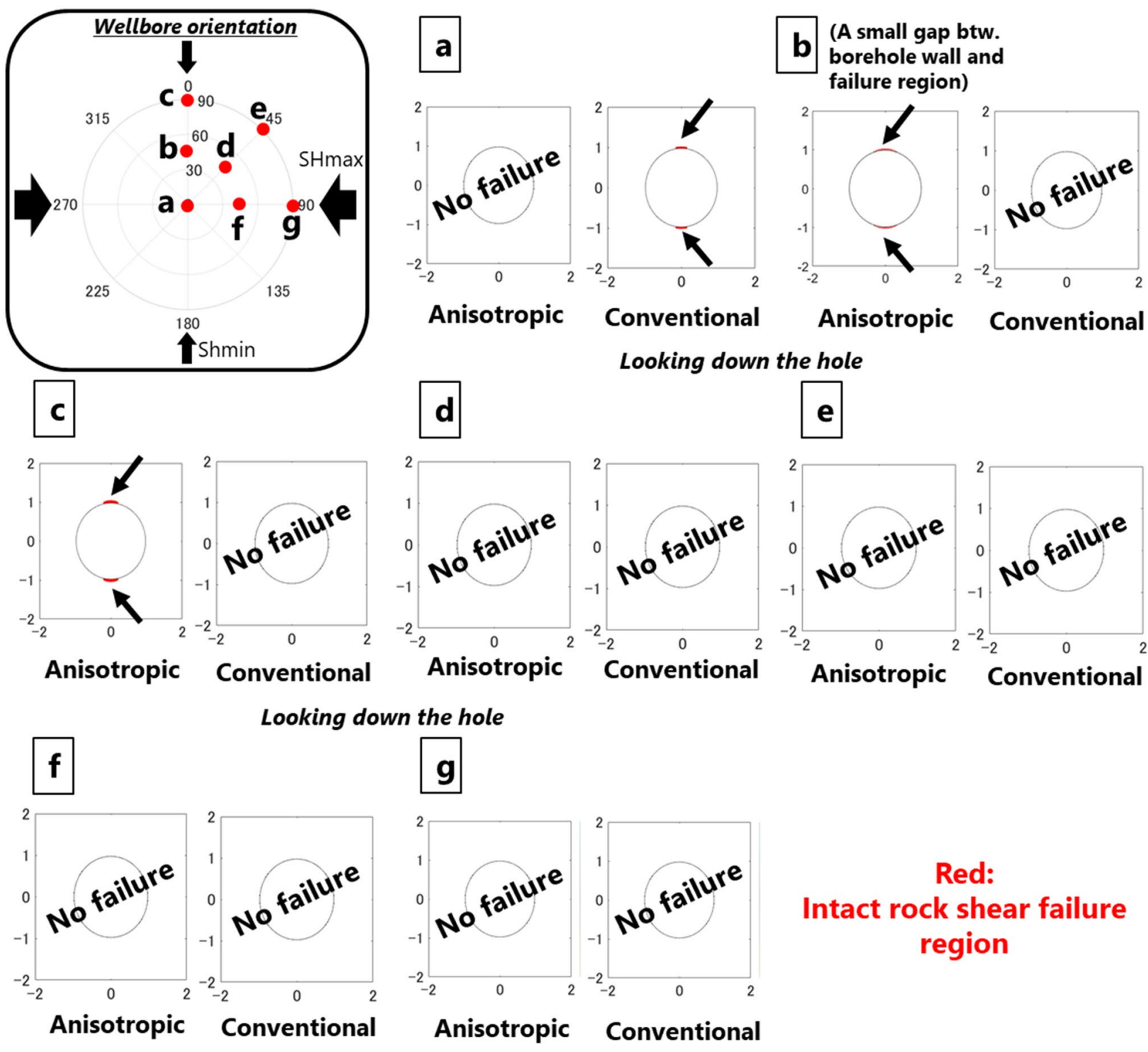

Fig. 4 A comparison of the predicted intact rock shear failure region based on the anisotropic approach (the Amadei solution +anisotropic poroelasticity) with that based on the conventional approach (the Kirsch solution + no induced pore pressure assumption)

Figures 4, 5 and 6 show a comparison of the predicted failure regions based on the anisotropic approach with that based on the conventional approach. Three different failure regions were predicted; (1) shear failure across the intact rock (Fig. 4), (2) slippage along the weak bedding planes (Fig. 5), and (3) tensile failure (tensile fracture + radial tensile failure, Fig. 6; radial tensile failures were identified when the angle between the minimum principal stress and radial directions is less than $10^{\circ}$ ). As shown in the figure, modelled failure regions are different, which is mainly caused by the induced pore pressure. The difference in the tensile failure regions are noticeable. For example, the conventional approach predicts tensile fracture at sides of borehole while the anisotropic approach predicts radial tensile failure at top and bottom of borehole for wellbore orientations a and $b$. For a well drilled in the maximum horizontal stress direction (orientation $\mathrm{g}$ ), the conventional approach predicts no failure but the anisotropic approach shows noticeable radial tensile failure at sides of borehole. The observations demonstrate that an unexpected wellbore failure may happen without consideration of elastic anisotropy.

Bedding plane failure regions strongly depend on wellbore orientation. For example, a horizontal well drilled in the maximum horizontal stress direction (orientation g) does not show bedding plane failures, while other two horizontal wells (orientations c and e) show noticeable 


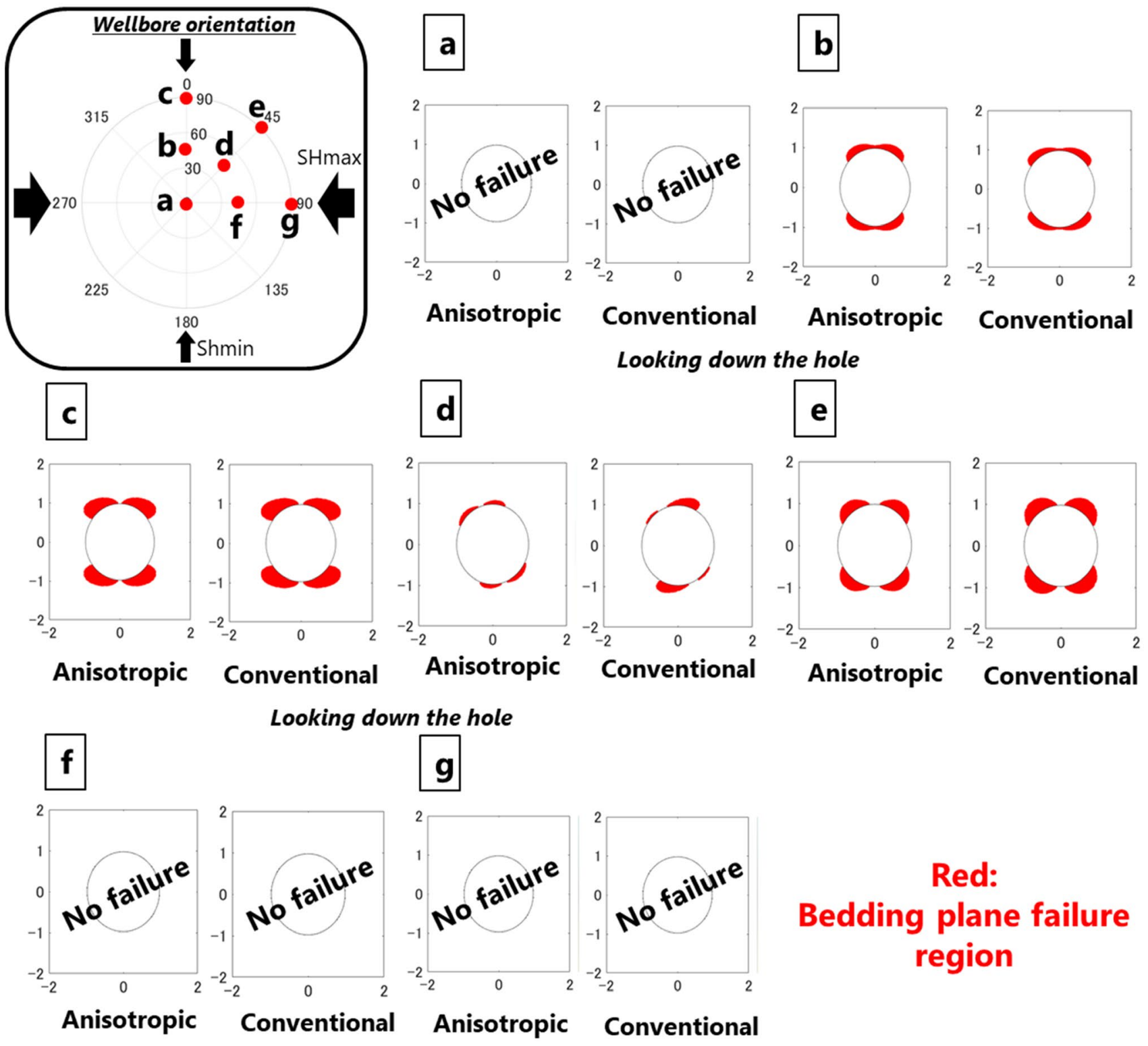

Fig. 5 A comparison of the predicted bedding plane failure region based on the anisotropic approach (the Amadei solution + anisotropic poroelasticity) with that based on the conventional approach (the Kirsch solution + no induced pore pressure assumption)

failures. Inclination of the bedding planes also affects the failure region (e.g., Deangeli and Omwanghe 2018). Insitu stress and bedding plane orientations should therefore be correctly accounted for to assess the wellbore stability. Note that wellbore orientation $\mathrm{c}$ is similar to one of cases considered in Deangeli and Omwanghe (2018). Although the elastic anisotropy and initial undrained pore pressure response are not considered by them, the failure regions predicted for the wellbore orientation are consistent with their results. Similarly, Fig. 5 indicates that the impact of elastic anisotropy on the bedding plane failure prediction is small, however, it actually depends on the wellbore orientation. For example, Fig. 7 shows a comparison of failure region for the wellbore orientation with inclination of $70^{\circ}$ and azimuth of $70^{\circ}$. The anisotropic approach shows smaller region of bedding plane failure mainly because of larger effective normal stress acting on the bedding plane at top and bottom of borehole than that based on the conventional approach. The larger effective normal stress is caused by pore pressure reduction at top and bottom of borehole (see the induced pore pressure in the figure). On the other hand, the anisotropic approach shows noticeable radial tensile failure at sides of borehole which is not predicted by the conventional approach. Predicted failure region is hence completely different in this case. 


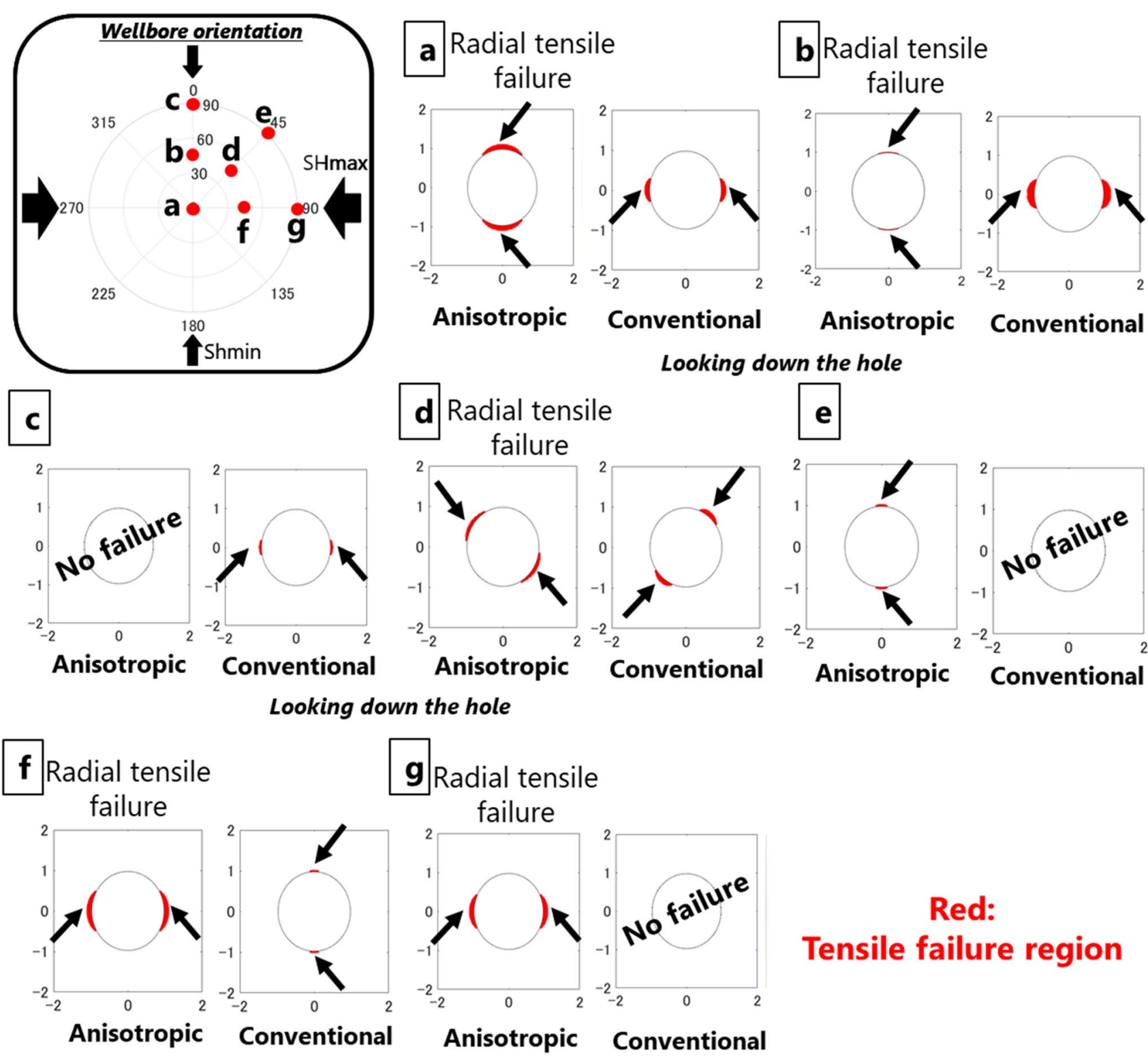

Fig. 6 A comparison of the predicted tensile failure region based on the anisotropic approach (the Amadei solution + anisotropic poroelasticity) with that based on the conventional approach (the Kirsch solution + no induced pore pressure assumption)

\section{Uncertainty of Input Parameters}

Several of the input parameters in wellbore stability analysis are uncertain. In general, subsurface stress measurements and pore pressure estimates in overburden formations are often absent and may have to be guessed based on regional experience. Core data on shale exist, but tests are time consuming, challenging and non-standard for most laboratories. In the present case, log data were available from the overburden. Sonic log data provide dynamic elastic moduli, which are known to be different from their static counterparts due to dispersion and strain dependence (Fjaer 2019). These moduli represent upper limits to undrained static stiffnesses and may provide information about shale strength through empirical correlations (Horsrud 2001).

Since this paper is focused on anisotropy, our discussion of uncertainty is related mainly to undrained rock anisotropy parameters and grain properties, which affect anisotropic Skempton's parameters estimation. The best way to minimize the uncertainty is obviously to measure the static elastic anisotropy parameters and Skempton's parameters [e.g. Holt et al. (2018a, b)]. If the measured Skempton's parameters were available from core studies, the estimation performed here would not be necessary. 

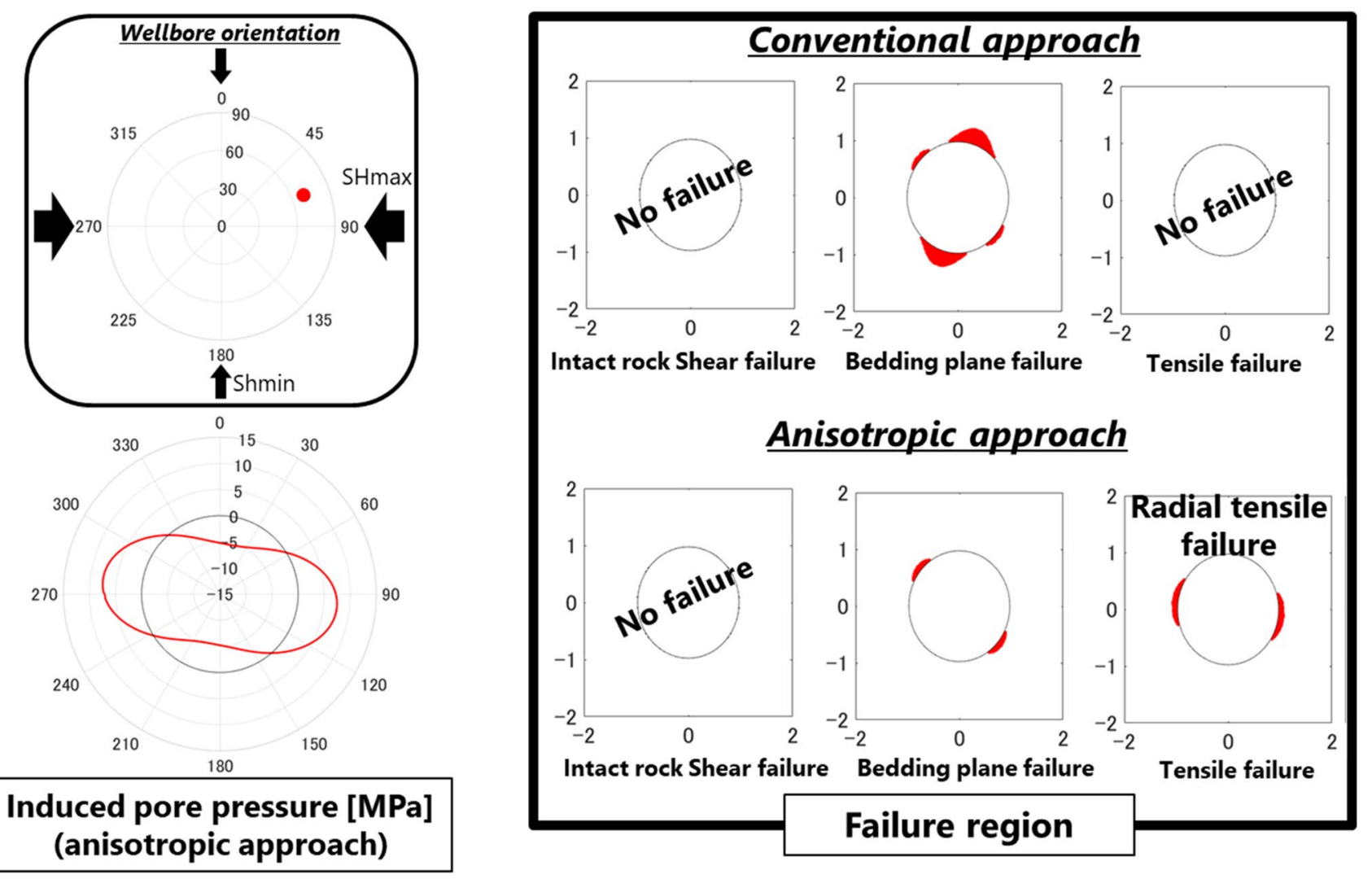

Fig. 7 Induced pore pressure based on the anisotropic approach (lower left) and a comparison of failure region based on the anisotropic approach with that based on the conventional approach (right) for the wellbore orientation with inclination of $70^{\circ}$ and azimuth of $70^{\circ}$

Table 3 Calculated Skempton's parameters and Biot coefficients with grain bulk modulus of 25 GPa and undrained anisotropy parameters delta of 0.2 and epsilon of 0.3

\begin{tabular}{ll}
\hline$B_{\mathrm{H}}$ & 0.07 \\
$B_{\mathrm{V}}$ & 1.52 \\
$A_{\mathrm{s}}(0)$ & 0.91 \\
$B_{\mathrm{S}}$ & 0.56 \\
$\alpha_{\mathrm{H}}$ & 0.58 \\
$\alpha_{\mathrm{V}}$ & 0.72 \\
\hline
\end{tabular}

\subsection{Undrained Rock Anisotropy Parameters}

As mentioned earlier, the undrained rock anisotropy parameters are estimated mainly from the seismic pre-stack depth migration velocity, which may contain error associated with imperfect data processing. Moreover, static elastic anisotropy could be different from the dynamic anisotropy. Larger anisotropy parameters (a delta of 0.2 and an epsilon of 0.3 ) were therefore tested. The same grain bulk modulus (25 GPa) was used. Resultant Skempton's parameters have larger anisotropy (larger $B_{\mathrm{V}}$ and smaller $B_{\mathrm{H}}$ ) than the aforementioned case as shown Table 3, as expected. Stress concentration for the larger undrained rock anisotropy case is shown in Fig. 8, indicating that the change in anisotropy parameters has only small impact. On the other hand, due to changes in Skempton's parameters, induced pore pressure shows noticeable changes (Fig. 9), especially for wells drilled in the minimum horizontal stress direction (orientations $b$ and $c$ ). This is mainly because of smaller Skempton's parameter $B_{\mathrm{H}}$; changes in stress parallel to bedding have only minor impact on the induced pore pressure. For example, large hoop stress at top and bottom of borehole for the orientation c gives only minor impact in this case. The change results in no intact rock shear failure for these orientations (Fig. 10). Moreover, smaller pore pressure decrease at top and bottom of borehole for the orientation $d$ and larger pore pressure increase at sides of borehole for the orientation e result in tensile fracture and radial tensile failure for these locations, respectively (Fig. 10). These failures are not predicted by the aforementioned case.

\subsection{Grain Modulus}

As mentioned earlier, the Skempton's parameters were estimated through the inverse anisotropic Gassmann's equation and anisotropic poroelasticity assuming elastically isotropic grains with a bulk modulus of $25 \mathrm{GPa}$. To see the impact of this assumption, two different scenarios were tested; (1) stiffer isotropic grain case and (2) anisotropic grain case. 


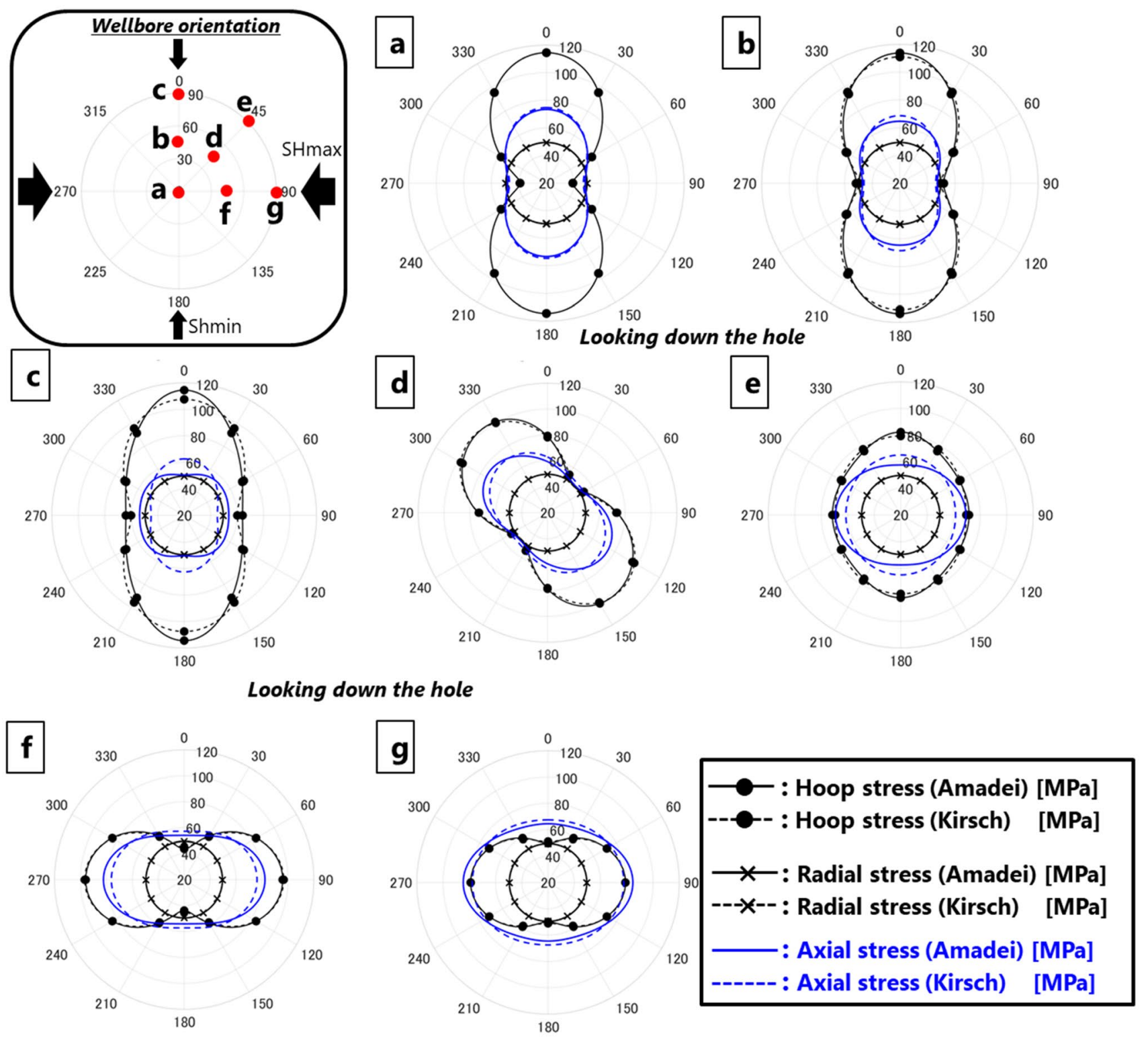

Fig. 8 A comparison of stress concentration at a circular borehole wall between the Amadei and Kirsch solutions for seven different wellbore orientations (larger undrained rock anisotropy was used for the Amadei solution)

A bulk modulus of $50 \mathrm{GPa}$ was used for the stiffer case while the best fitting TI approximations of first-principal calculations by Militzer et al. (2011) for dry muscovite (Sayers and den Boer 2016; $C_{11}=C_{22}=181.3, C_{13}=24.8$, $C_{33}=60.1, C_{44}=C_{55}=20.3, C_{66}=66.3$, all in units of GPa) were used for the anisotropic grain case. The same undrained rock anisotropy parameters were used (a delta of 0.12 and an epsilon of 0.21 ). Table 4 shows the corresponding Skempton's parameters and Biot's coefficients. The stiffer grain case shows larger Skempton's parameters while the anisotropic grain case shows less anisotropy $\left(B_{\mathrm{H}}\right.$ is larger but $B_{\mathrm{V}}$ is slightly smaller) in comparison with the result shown in Table 2. Figures 11 and 12 show the induced pore pressure for the stiffer and the anisotropic grain case, respectively. Both cases give larger pore pressure change for most of borehole orientations than the aforementioned cases. This is because of larger Skempton's parameters. The larger pore pressure change results in larger radial tensile failures (Fig. 13).

\subsection{Comparison with Existing Measurements}

Estimated Skempton's parameters are compared with existing measurement results. Holt et al. (2018b) provided measured values for three shales, while Lozovyi and Bauer (2019) gave results for a shaly facies of Opalinus Clay. Skempton's 


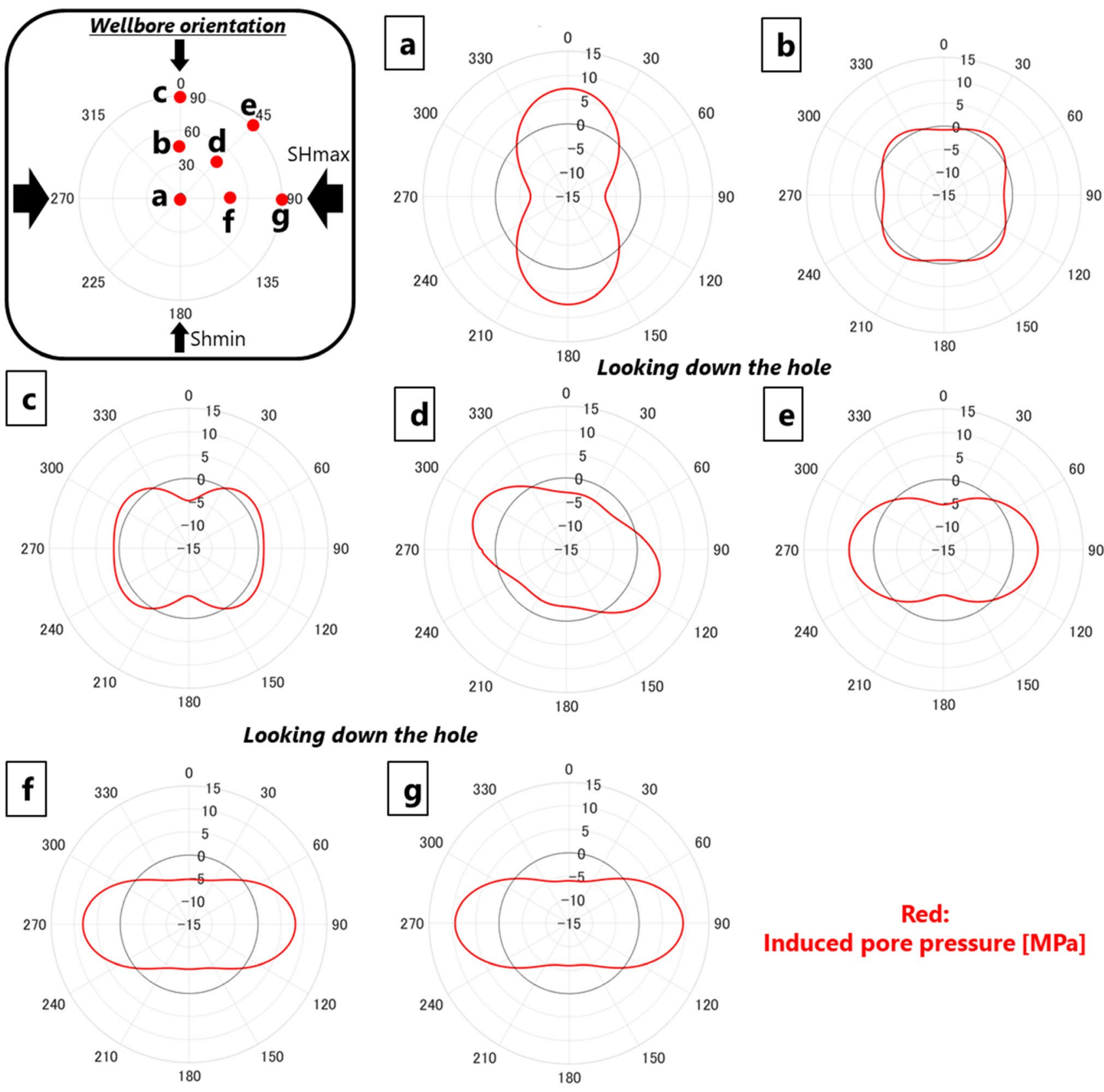

Fig. 9 Induced pore pressure at a circular borehole wall based on the anisotropic approach (the Amadei solution + anisotropic poroelasticity) for the larger undrained rock anisotropy case

parameters of the stiffer grain case are consistent with shale "B" and "M" of Holt et al. (2018b) and the shaly facies of Lozovyi and Bauer (2019), while the anisotropic grain case is consistent with shale " $\mathrm{D}$ " of Holt et al. (2018b). Those tested parameters are therefore realistic values. Skempton's parameters for other cases (those listed in Table 2 and 3) are different from these measurement results, however, experimental results are limited and those cases are based on realistic grain bulk modulus and undrained rock anisotropy parameters; those cases are therefore considered as possible cases here. It should be noted that Skempton parameters based on elasticity is not valid close to failure since it is affected by the transition from elastic to plastic deformation. This is a topic for further investigation. The current analysis is however expected to be applicable to initial state. 


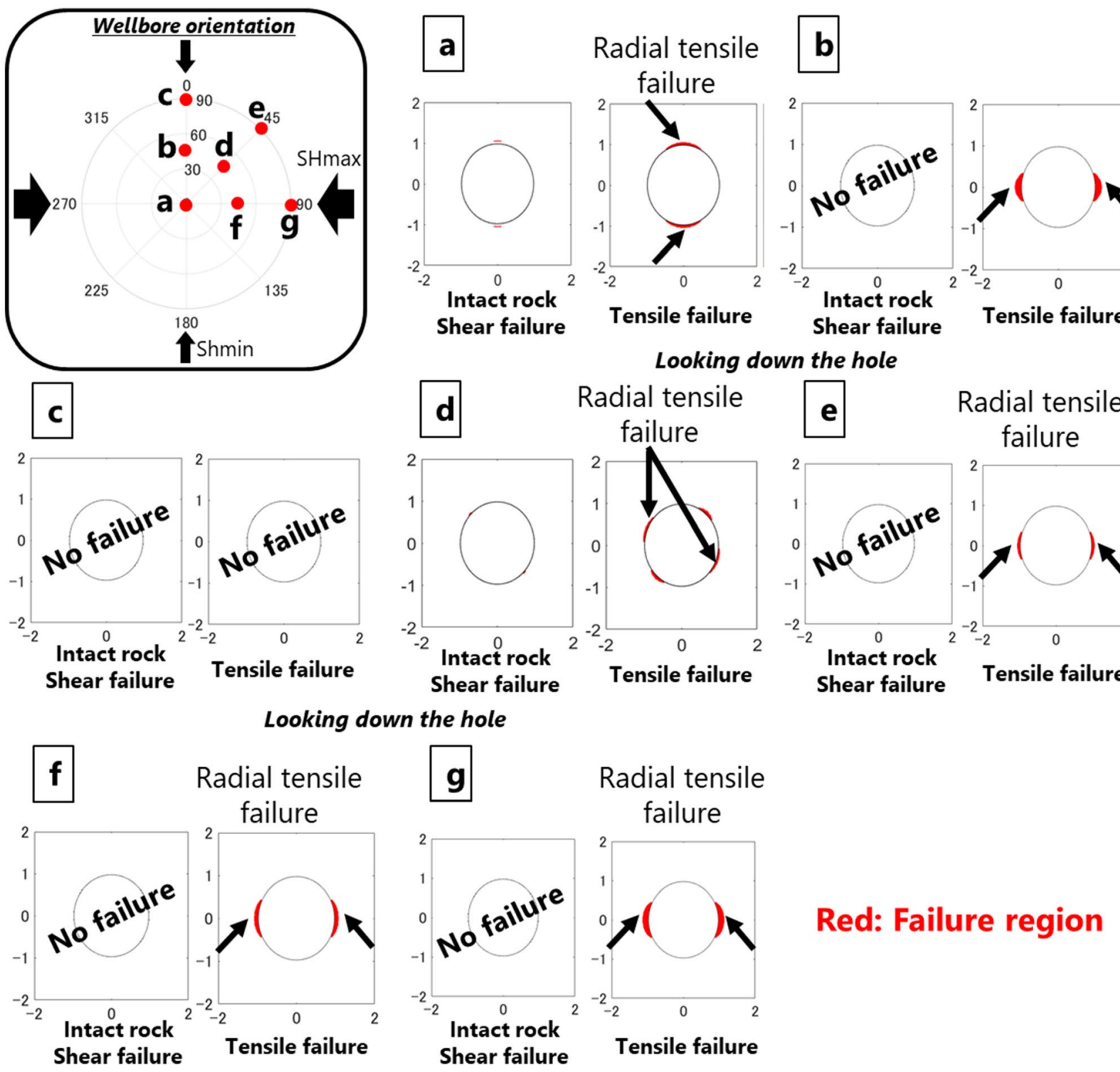

Fig. 10 Predicted intact rock shear failure and tensile failure regions for the larger undrained rock anisotropy cases

Table 4 Calculated Skempton's parameters and Biot coefficients for the stiffer and anisotropic grain cases

\begin{tabular}{lll}
\hline & $\begin{array}{l}\text { Stiffer } \\
\text { grain } \\
\text { case }\end{array}$ & $\begin{array}{l}\text { Anisotropic } \\
\text { grain case }\end{array}$ \\
\hline$B_{\mathrm{H}}$ & 0.66 & 0.82 \\
$B_{\mathrm{V}}$ & 1.36 & 1.04 \\
$A_{\mathrm{s}}(0)$ & 0.51 & 0.39 \\
$B_{\mathrm{s}}$ & 0.89 & 0.89 \\
$\alpha_{\mathrm{H}}$ & 0.95 & 0.99 \\
$\alpha_{\mathrm{V}}$ & 0.98 & 0.94 \\
\hline
\end{tabular}

\section{Discussion}

It was demonstrated that the anisotropic approach gives considerably different failure regions and modes in comparison with the conventional approach. For example, vertical wells and horizontal wells in the maximum horizontal stress direction show noticeable radial tensile failures which are not predicted by the conventional approach as shown in Fig. 6. The predictions by the anisotropic approach are apparently consistent with observations in 


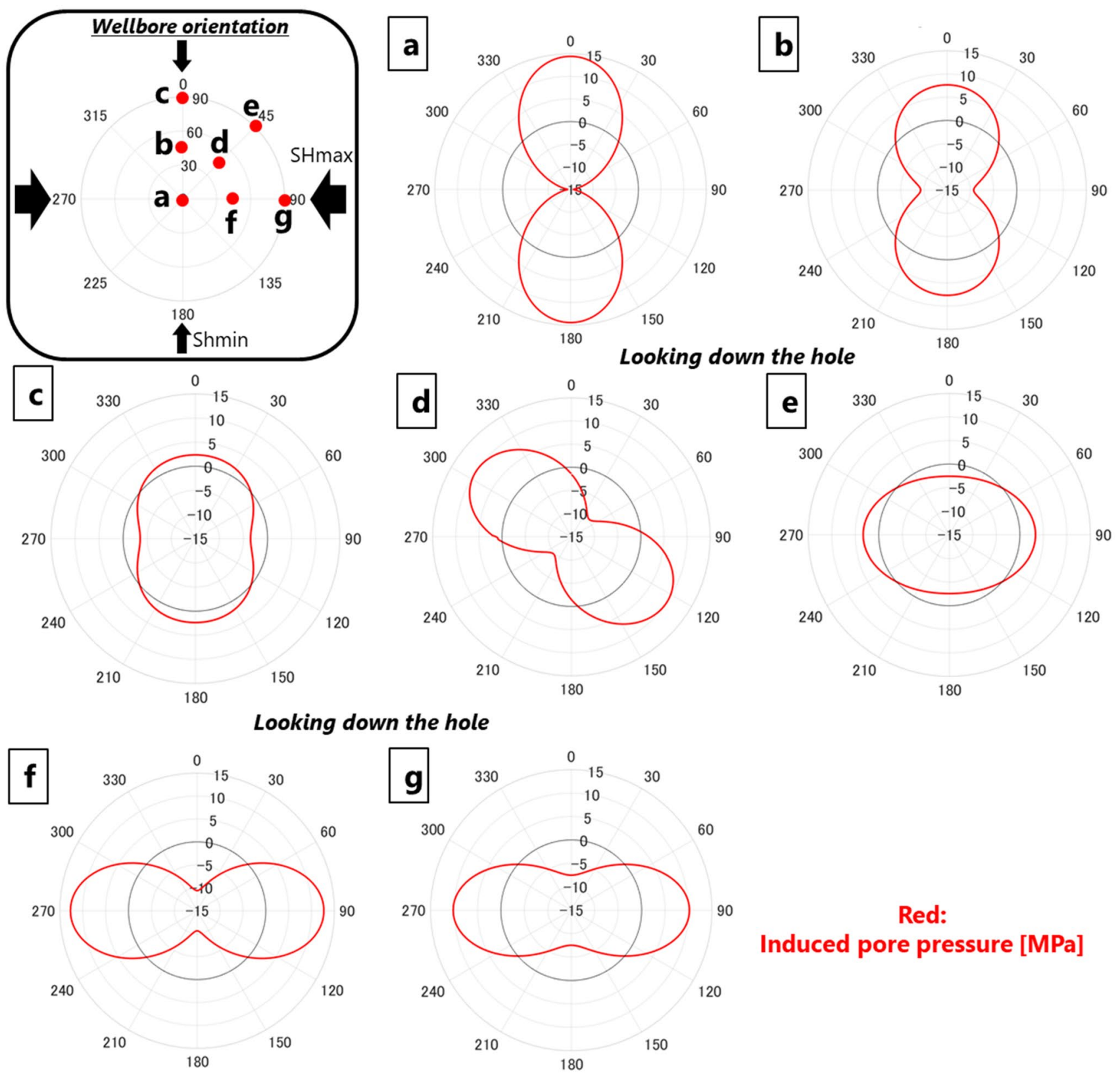

Fig. 11 Induced pore pressure at a circular borehole wall based on the anisotropic approach (the Amadei solution + anisotropic poroelasticity) for the stiffer grain case

the field: (1) highly inclined wells drilled in a direction of the maximum horizontal stress tend to show wellbore failures at sides of borehole in shale sections. Example of computed radius data based on Stabilized Azimuthal Density Neutron image data (SADNTM) collected in one of highly inclined wells in the maximum horizontal stress direction is shown in Fig. 14. (2) Splintery cavings were observed from breakouts in shale section in the existing vertical well (Fig. 15); such splintery cavings are produced 


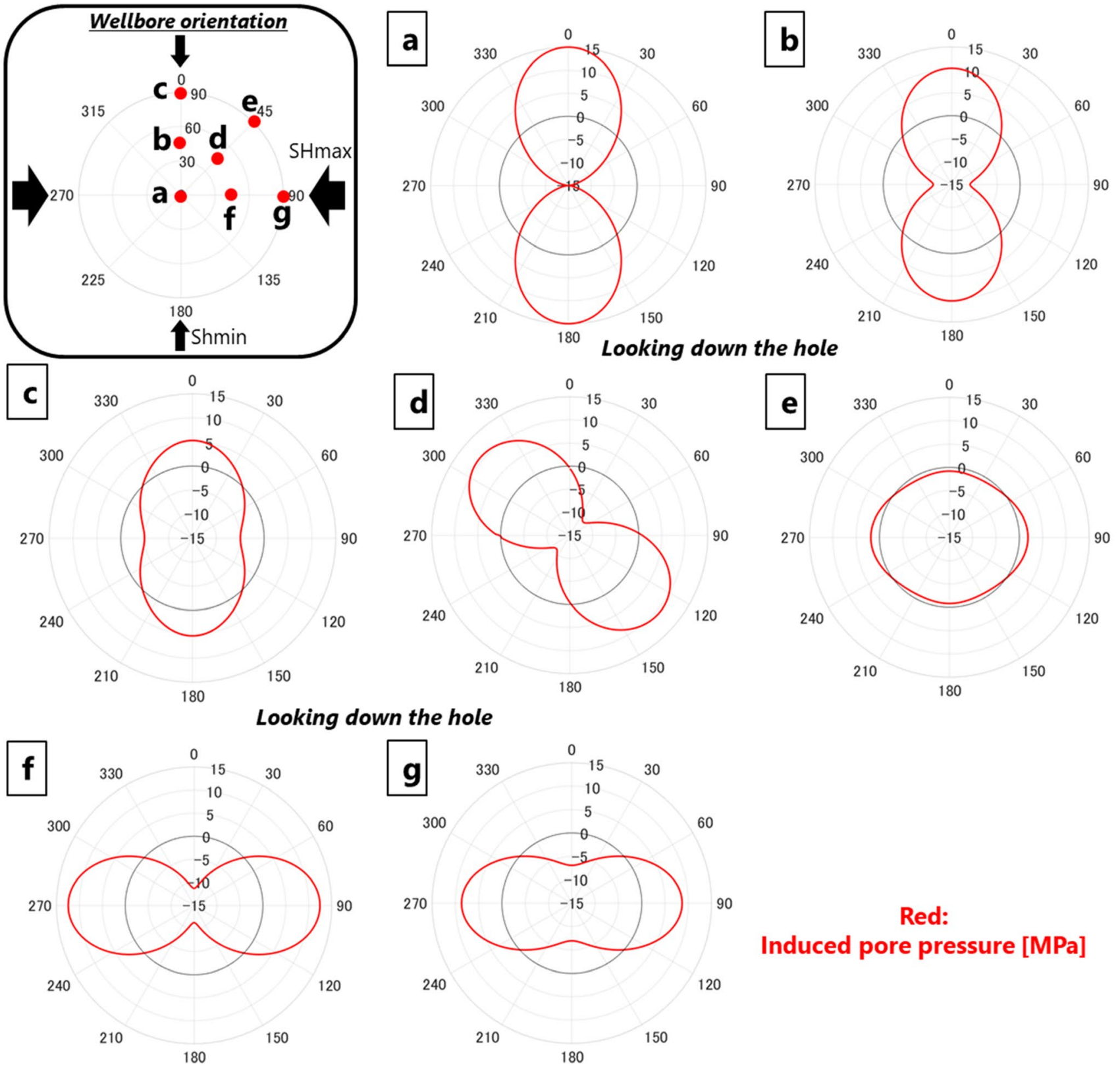

Fig. 12 Induced pore pressure at a circular borehole wall based on the anisotropic approach (the Amadei solution + anisotropic poroelasticity) for the anisotropic grain case

in zones where tensile failures occur throughout the wellbore circumference (Skea et al. 2018).

The difference between the two approaches is largely due to the induced pore pressure, which is associated with anisotropy in Skempton's parameters and in-situ stress state. Induced pore pressure is less sensitive to changes in stress parallel to bedding for transverse isotropic rocks such as shales. If in situ stress state is a strike-slip regime with significant stress anisotropy, as shown here, horizontal wells drilled in the maximum horizontal stress direction will show completely different induced pore pressure compared with horizontal wells drilled in the minimum horizontal stress direction as shown in Fig. 2. Horizontal wells in the maximum horizontal stress direction, which is a preferable drilling direction in a strike-slip regime as opposed to normal fault stress states, will show large pore pressure increase at sides of borehole, which may trigger radial tensile failure. If in situ stress state is a normal faulting regime, horizontal wells in shale are expected to have increased pore pressure at sides of borehole regardless of direction. For a reverse faulting regime, on the other hand, smaller induced pore pressure is expected for horizontal wells in shale. 

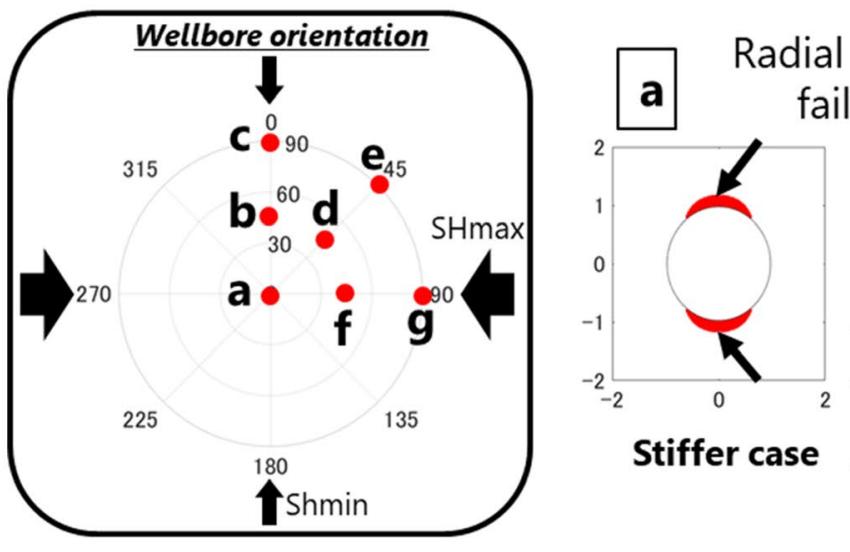

fal tensile failure
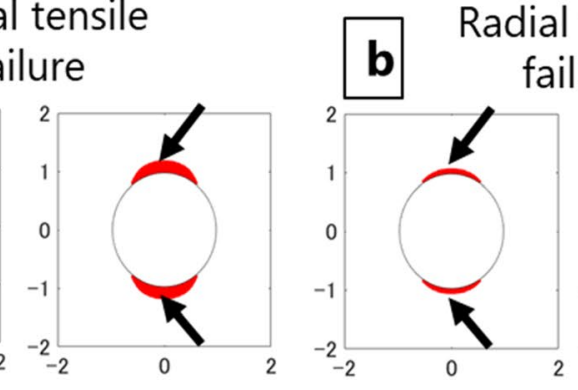
failure
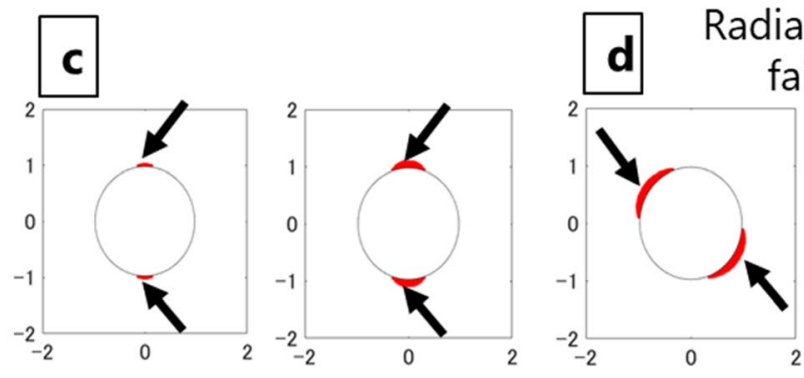

Anisotropic case

Stiffer case Looking down the hole

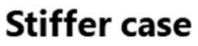

Anisotropic case

Stiffer case

failure

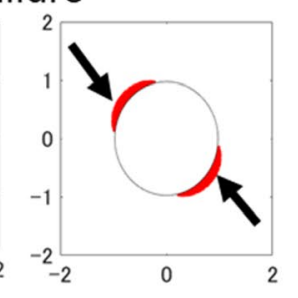

Anisotropic case

Looking down the hole

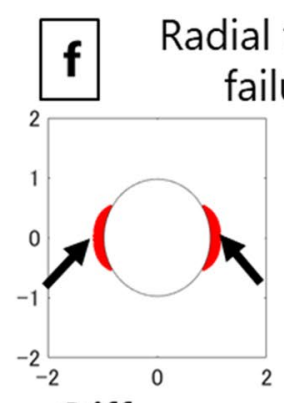

Stiffer case tensile

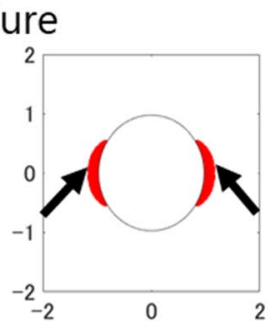

Anisotropic case

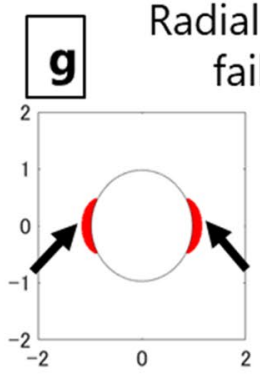

Stiffer case ial tensile

ailure

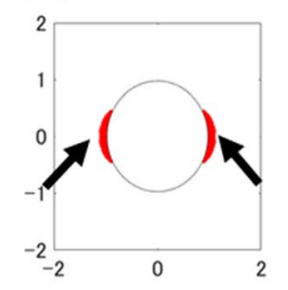

Anisotropic case

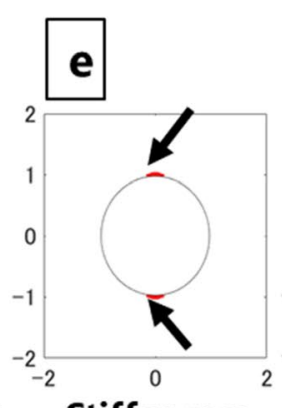

Stiffer case

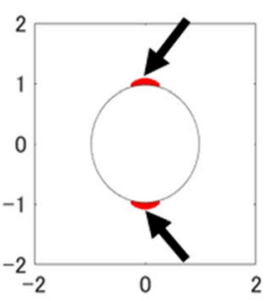

Anisotropic case

Fig. 13 Predicted tensile failure regions for the stiffer and the anisotropic grain cases

To see the influence of anisotropy on induced pore pressure, induced pore pressure was calculated by setting anisotropy parameters zero for both stress and Skempton's parameters calculation (hence equivalent to use Kirsch solution and isotropic elasticity) and compared with that based on the anisotropic approach shown in Fig. 2. The isotropic assumption gives Skempton's $B$ parameter of 0.85 in this case (Skempton's $A_{\mathrm{s}}$ parameters is $1 / 3$ for isotropic elasticity). The results are shown in Fig. 16. The anisotropic case shows smaller pore pressure change than the isotropic case for wellbore orientations $b$ and $c$, because of anisotropy in Skempton's parameters; induced pore pressure is less sensitive to changes in stress parallel to bedding. Neglecting the influence of anisotropy can therefore lead to incorrect failure predictions.

The impact on mud weights should be mentioned. Figure 17 shows a comparison of estimated maximum and minimum mud weights for wells drilled in the maximum horizontal stress direction based on the anisotropic approach with that based on the conventional approach. The same input parameters are used (Table 1). To show a practical mud weight window, minimum mud weights related to intact rock shear failure and radial tensile failure are calculated to prevent specified failure width $\left(90^{\circ}\right.$ wide for vertical wells and $30^{\circ}$ wide for horizontal wells; those values were linearly interpolated for other inclinations), and plotted in the lower 
Fig. 14 Computed radius data based on SADN image density data in shale section in highly inclined well drilled in the maximum horizontal stress direction
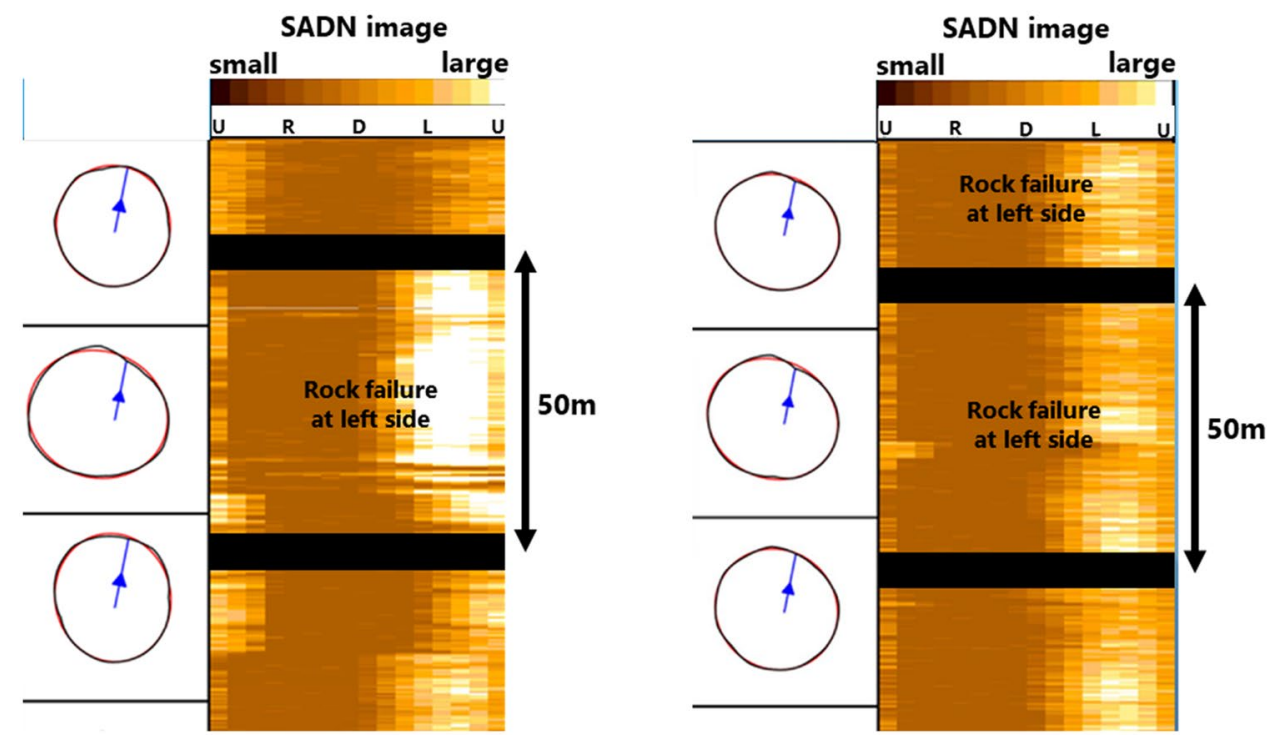

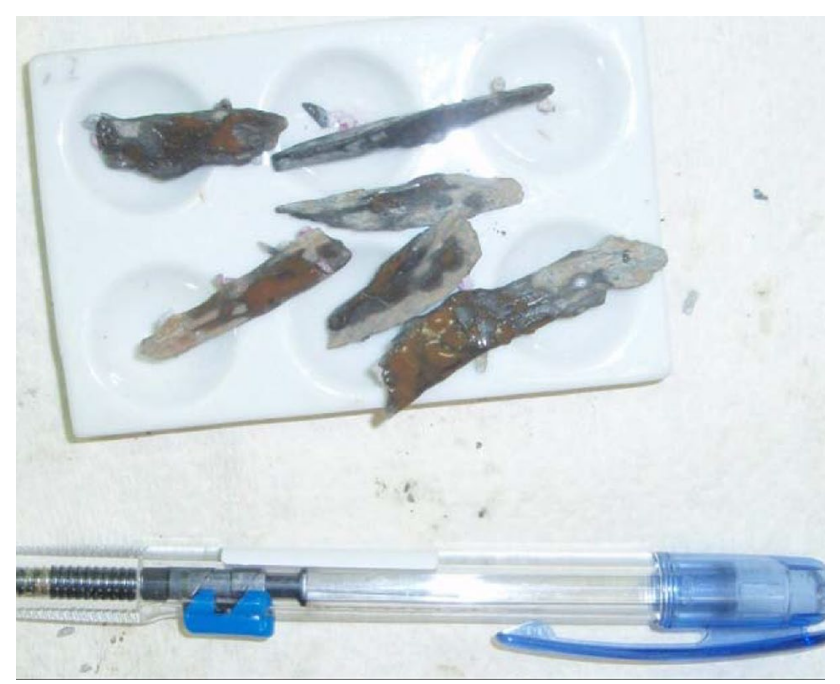

Fig. 15 Splintery cavings from breakouts in a thick shale section in the existing vertical well

figure. As shown in the figure, the predicted mud weights are completely different. Key findings are as follows:

- The anisotropic approach does not predict shear failures across the intact rock at borehole wall in this case. Instead, radial tensile failures are predicted. This is because induced pore pressure makes the effective radial stress smaller than the tensile strength at angular borehole positions with high hoop stress when low mud weight is used. Tensile strength needs to be higher to have intact rock shear failures. Moreover, the minimum mud weight required to prevent the radial tensile failure is larger than that to prevent the intact shear rock failures predicted by the conventional approach.

- The anisotropic approach gives significantly larger maximum mud weight to prevent tensile fracture for low inclinations because of reduction in pore pressure at wellbore positions with small hoop stress (see Figs. 1 and 2).

This results in wider mud weight window for low inclinations if we accept some radial tensile failures (the conventional approach appears to give no mud weight window for low inclinations. However, drilling would be possible with mud weight lower than the minimum horizontal stress since fracture growth is not expected even if fractures are formed). For highly inclined wells $\left(>70^{\circ}\right)$, on the other hand, the conventional approach gives wider mud weight window without any failure. The anisotropic approach gives narrower window and we have to accept some radial tensile failures associated with pore pressure increase at sides of borehole. In summary, the anisotropic approach makes drilling easier for low inclination wells and difficult for highly inclined wells in this case. The observation for highly inclined wells is also applicable to normal fault stress states.

\section{Conclusions}

Anisotropic wellbore stability analysis was performed using the Amadei solutions and the Skempton's parameters based on anisotropic poroelasticity and compared with the conventional approach. The comparison revealed that induced pore pressure has significant impact on the results. Induced pore pressure depends on anisotropy in Skempton's parameters, 


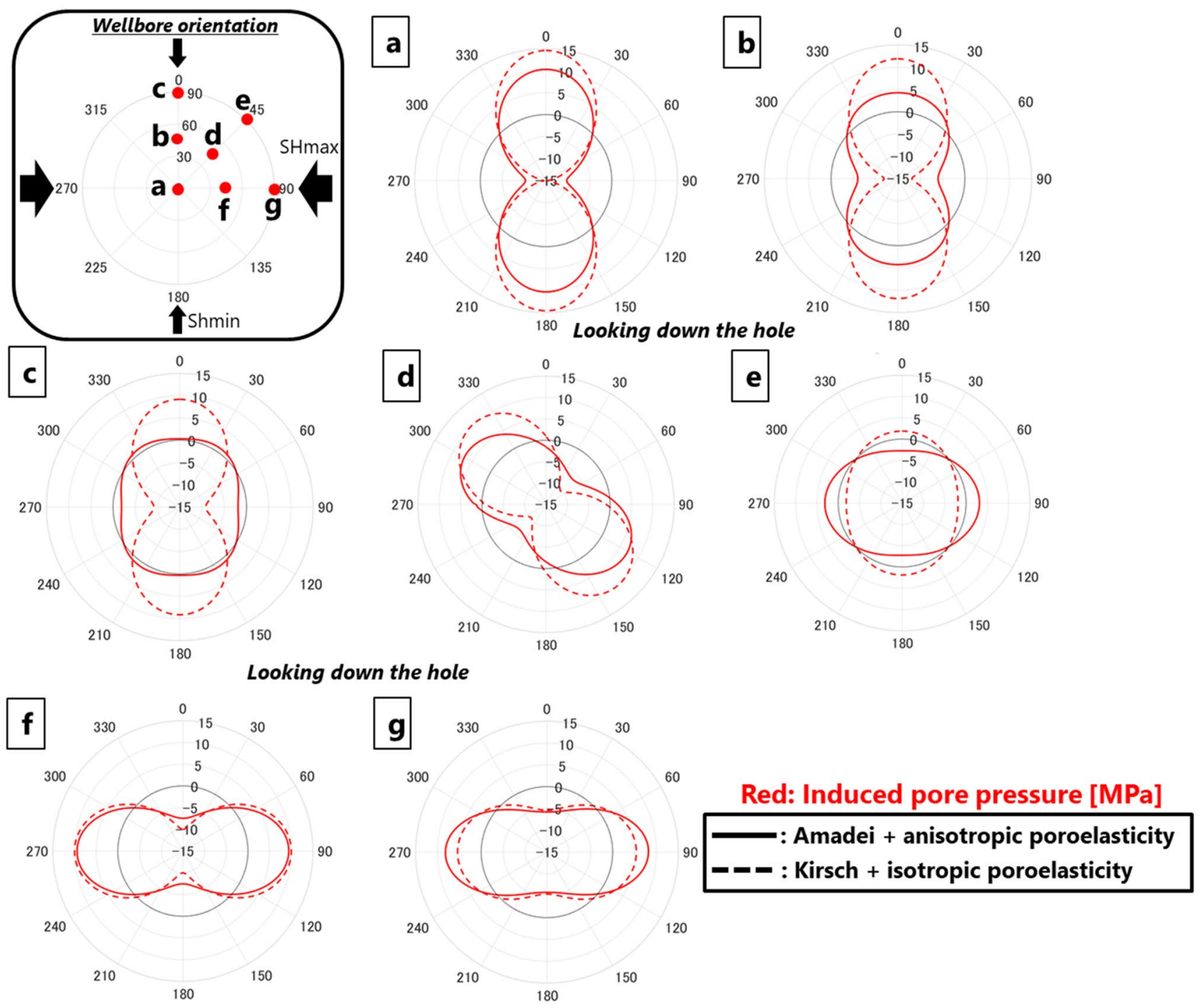

Fig. 16 A comparison of induced pore pressure at a circular borehole wall based on the anisotropic approach (the Amadei solution + anisotropic poroelasticity) with that based on the isotropic approach (the Kirsch solution + isotropic poroelasticity)

in situ stress state and wellbore orientation. For example, highly inclined wells in a normal faulting regime and that in the maximum horizontal stress direction in a strike-slip regime tend to show large pore pressure increase at sides of borehole and reduction in pore pressure at top and bottom of borehole. The pore pressure increase may trigger radial tensile failures which are not predicted by the conventional approach. The pore pressure reduction, on the other hand, may prevent tensile fractures predicted by the conventional approach. Mud weight window is also affected by elastic anisotropy. The anisotropic approach tends to give wider mud weight window for low inclination wells in stress states with large horizontal stress anisotropy, mainly because of pore pressure reduction at wellbore positions with small hoop stress (i.e., increase in maximum mud weight to prevent tensile fracture). In contrast, it gives narrower mud weight window for highly inclined wells in a normal faulting stress state and that in a preferable direction (i.e. maximum horizontal stress direction) in a strike-slip stress state with large stress anisotropy, due to pore pressure increase at sides of borehole. Uncertainty study revealed that changes in input parameters have certain impact on the Skempton's parameters, which should be correctly accounted for to predict induced pore pressure. 


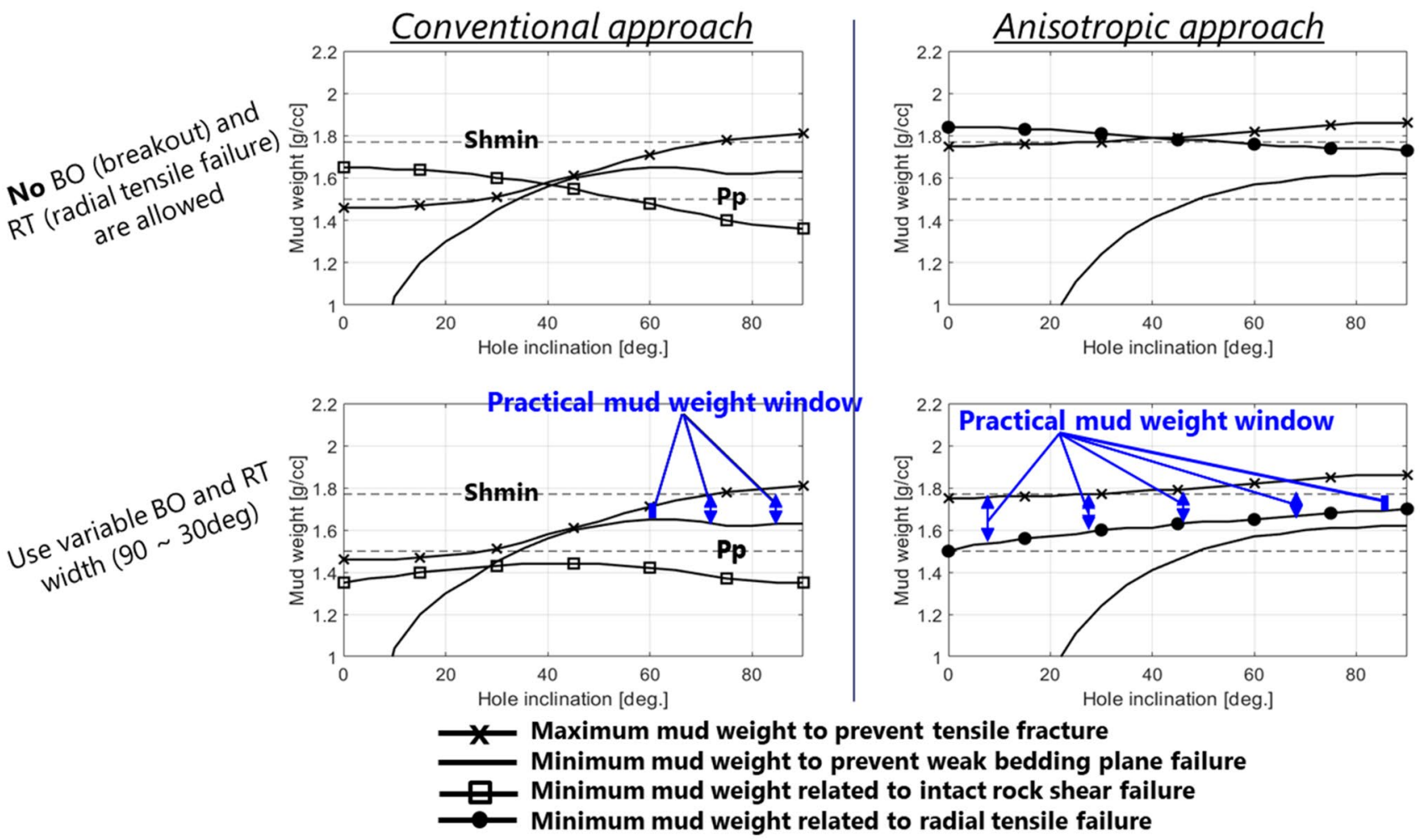

Fig. 17 A comparison of maximum/minimum mud weights for wells drilled in the maximum horizontal stress direction based on the conventional approach (left) with that based on the anisotropic approach (right). Upper figure shows minimum mud weights to prevent any intact rock shear failure and radial tensile failure, while lower figure shows that to prevent specified failure width $\left(90^{\circ}\right.$ wide for vertical wells and $30^{\circ}$ wide for horizontal wells; those values were interpolated for other inclinations). The minimum horizontal stress and pore pressure (before drill-out) are shown as black dashed lines

Acknowledgements We would like to thank INPEX management and all joint venture participants for allowing us to publish this work.

Funding Open Access funding provided by NTNU Norwegian University of Science and Technology (incl St. Olavs Hospital - Trondheim University Hospital).

Open Access This article is licensed under a Creative Commons Attribution 4.0 International License, which permits use, sharing, adaptation, distribution and reproduction in any medium or format, as long as you give appropriate credit to the original author(s) and the source, provide a link to the Creative Commons licence, and indicate if changes were made. The images or other third party material in this article are included in the article's Creative Commons licence, unless indicated otherwise in a credit line to the material. If material is not included in the article's Creative Commons licence and your intended use is not permitted by statutory regulation or exceeds the permitted use, you will need to obtain permission directly from the copyright holder. To view a copy of this licence, visit http://creativecommons.org/licenses/by/4.0/.

\section{References}

Abousleiman Y, Cui L (1998) Poroelastic solutions in transversely isotropic media for wellbore and cylinder. Int J Solids Struct $35: 4905-4929$
Abousleiman Y, Ekbote S (2005) Solutions for the inclined borehole in a porothermoelastic transversely isotropic medium. J Appl Mech $72: 102-114$

Amadei B (1983) Rock anisotropy and the theory of stress measurements. Springer Science and Business Media, Berlin

Asaka M, Sekine T, Furuya K (2016) Geologic cause of seismic anisotropy: a case study from offshore Western Australia. Lead Edge 35:662-668

Batzle M, Wang Z (1992) Seismic properties of pore fluids. Geophysics 57:1396-1408

Bouteca M, Gueguen Y (1999) Mechanical properties of rocks: pore pressure and scale effects. Oil Gas Sci Technol Rev IFP 54:703-714

Castagna JP, Batzle ML, Kan TK (1993) Rock physics-the link between rock properties and AVO response: in offset-dependent reflectivity - theory and practice of AVO analysis. Investigations in geophysics, vol 8. Society of Exploration Geophysicists, Tulsa, pp 135-171

Cheng AH-D (1997) Material coefficients of anisotropic poroelasticity. Int J Rock Mech Min Sci 34:199-205

Cornet F, Fairhurst C (1974) Influence of pore pressure on the deformation behavior of saturated rocks. In: Proceedings of 3rd Congr. ISRM, Denver

Deangeli C, Omwanghe OO (2018) Prediction of mud pressure for the stability of wellbores drilled in transversely isotopric rocks. Energies 11:1944 
Ekbote S, Abousleiman Y (2006) Porochemoelastic solution for an inclined borehole in a transversely isotropic formation. J Eng Mech 132:754-763

Fang X (2018) A revisit of the Lekhnitskii-Amadei solution for borehole stress calculation in tilted transversely isotorpic media. Int J Rock Mech Min Sci 104:113-118

Fjaer E (2019) Relations between static and dynamic moduli of sedimentary rocks. Geophys Prospect 67:128-139

Fjaer E, Holt RM, Horsrud P, Raaen AM, Risnes R (2008) Petroleum related rock mechanics, 2nd edn. Elsevier, Amsterdam, p 491

Gao J, Deng J, Lan K, Song Z, Feng Y, Chang L (2017) A porothermoelastic solution for the inclined borehole in a transversely isotropic medium subjected to thermal osmosis and thermal filtration effects. Geothermics 64:114-134

Gao J, Deng J, Lan K, Feng Y, Zhang W, Wang H (2017) Porothermoelastic effect on wellbore stability in transversely isotropic medium subjected to local thermal non-equilibrium. Int J Rock Mech Min Sci 96:66-84

Ghassemi A, Diek A (2002) Porothermoelasticity for swelling shales. J Petrol Sci Eng 34:123-135

Ghassemi A, Diek A (2003) Linear chemo-poroelasticity for swelling shales: theory and application. J Petrol Sci Eng 38:199-212

Hiramatsu Y, Oka Y (1962) Analysis of stress around a circular shaft or drift excavated in ground in a three dimentional stress state. $\mathrm{J}$ Min Metall Inst Jpn 78:93-98

Holt RM, Bakk A, Bauer A (2018a) Anisotropic poroelasticity-does it apply to shale?. In: 88th Annual International Meeting, SEG, expanded abstracts, pp 3512-3516

Holt RM, Bauer A, Bakk A (2018) Stress-path-dependent velocities in shales: Impact of 4D seismic interpretation. Geophysics 83:MR353-MR367

Horsrud P (2001) Estimating mechanical properties of shale from empirical correlations. SPE Drill Complet 16:68-73

Jaeger JC, Cook NGW (1979) Fundamental of rock mechanics, 3rd edn. Chapman and Hall, London

Kanfar MF, Chen Z, Rahman SS (2015a) Risk-controlled wellbore stability analysis in anisotropic formations. J Petrol Sci Eng 134:214-222

Kanfar MF, Chen Z, Rahman SS (2015b) Effect of material anisotropy on time-dependent wellbore stability. Int J Rock Mech Min Sci 78:36-45

Kanfar MF, Chen Z, Rahman SS (2016) Fully coupled 3D anisotropic conductive-convective porothermoelasticity modeling for inclined boreholes. Geothermics 61:135-148

Kanfar MF, Chen Z, Rahman SS (2017) Analyzing wellbore stability in chemically-active anisotropic formations under thermal, hydraulic, mechanical and chemical loadings. J Nat Gas Sci Eng 41:93-111
Karpfinger F, Prioul R, Gaede O, Jocker J (2011) Revisiting borehole stresses in anisotorpic elastic media: comparison of analytical versus numerical solutions. In: Proceedings of the 45th US rock mechanics/geomechanics symposium-ARMA. American Rock Mechanics Association

Kirsch EG (1898) Die Theorie der Elastizitat und die Bedurfnisse der Festigkeitslehre. Zeitschrift des Vereines deutscher Ingenieure 42:797-807

Lee H, Ong SH, Azeemuddin M, Goodman H (2012) A wellbore stability model for formations with anisotropic rock strengths. J Petrol Sci Eng 96:109-119

Lekhnitskii SG (1963) Theory of the elasticity of anisotopric bodies. Holden-Day, Toronto

Lozovyi S, Bauer A (2019) Static and dynamic stiffness measurements with Opalinus clay. Geophys Prospect 67:997-1019

Mavko G, Mukerji T, Dvorkin J (2009) The rock physics handbook, 2nd edn. Cambridge University Press, Cambridge

Militzer B, Wenk H-R, Stackhouse S, Stxrude L (2011) First-principles calculation of the elastic moduli of sheet silicates and their application to shale anisotropy. Am Miner 96:125-137

Norris AN, Sinha BK (1993) Weak elastic anisotropy and the tube wave. Geophysics 58:1091-1098

Raaen AM, Larsen I, Fjaer E, Holt RM (2019) Pore pressure reponse in rock: Implications of tensorial Skempton B in an anisotorpic formation. In: Proceedings of the 53rd US rock mechanics/ geomechanics symposium-ARMA. American Rock Mechanics Association

Sayers CM, den Boer LD (2016) The elastic anisotropy of clay minerals. Geophysics 81:C193-C203

Skea C, Rezagholilou A, Far PB, Gholami R, Sarmadivleh M (2018) An approach for wellbore failure analysis using rock cavings and image processing. J Rock Mech Geotech Eng 10:865-878

Soler JM (2001) The effect of coupled transport phenomena in the Opalinus clay and implications for radionuclide transport. $\mathbf{J}$ Contam Hydrol 53:63-84

Szewczyk D, Bauer A, Holt RM (2018) Stress-dependent elastic properties of shales-laboratory experiments at seismic and ultrasonic frequencies. Geophys J Int 212:189-210

Thomsen L (1986) Weak elastic anisotropy. Geophysics 51:1954-1966

Vernik L, Nur A (1992) Ultrasonic velocity and anisotropy of hydrocarbon source rocks. Geophysics 57:727-735

Wang Z (2002) Seismic anisotropy in sedimentary rocks, part 2: laboratory data. Geophysics 67:1423-1440

Publisher's Note Springer Nature remains neutral with regard to jurisdictional claims in published maps and institutional affiliations. 